Issued by Sandia National Laboratories, operated for the United States Department of Energy by Sandia Corporation.

NOTICE: This report was prepared as an account of work sponsored by an agency of the United States Government. Neither the United States Government, nor any agency thereof, nor any of their employees, nor any of their contractors, subcontractors, or their employees, make any warranty, express or implied, or assume any legal liability or responsibility for the accuracy, completeness, or usefulness of any information, apparatus, product, or process disclosed, or represent that its use would not infringe privately owned rights. Reference herein to any specific commercial product, process, or service by trade name, trademark, manufacturer, or otherwise, does not necessarily constitute or imply its endorsement, recommendation, or favoring by the United States Government, any agency thereof, or any of their contractors or subcontractors. The views and opinions expressed herein do not necessarily state or reflect those of the United States Government, any agency thereof, or any of their contractors.

Printed in the United States of America. This report has been reproduced directly from the best available copy.

Available to DOE and DOE contractors from Office of Scientific and Technical Information P.O. Box 62

Oak Ridge, TN 37831

Prices available from (703) 605-6000

Web site: http://www.ntis.gov/ordering.htm

Available to the public from National Technical Information Service U.S. Department of Commerce 5285 Port Royal Rd

Springfield, VA 22161

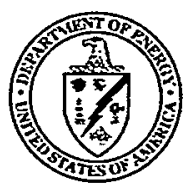




\section{DISCLAIMER}

Portions of this document may be illegible in electronic image products. Images are produced from the best available original document. 
SAND99-3061

Unlimited Release

Reprinted March 2000

The only change to this document is the addition of the appendices

\title{
Surfactant-Modified Diffusion on Transition-Metal Surfaces
}

\author{
Peter J. Feibelman and Gary L. Kellogg \\ Surface and Interface Sciences Department \\ Sandia National Laboratories \\ Albuquerque, NM 87185-1421
}

\begin{abstract}
Wanting to convert surface impurities from a nuisance to a systematically applicable nano-fabrication tool, we have sought to understand how such impurities affect selfdiffusion on transition-metal surfaces. Our field-ion microscope experiments reveal that in the presence of surface hydrogen, self-diffusion on $\mathrm{Rh}(100)$ is promoted, while on $\mathrm{Pt}(100)$, not only is it inhibited, but its mechanism changes. First-principles calculations aimed at learning how oxygen fosters perfect layerwise growth on a growing $\operatorname{Pt}(111)$ crystal contradict the idea in the literature that it does so by directly promoting transport over Pt island boundaries. The discovery that its real effect is to burn off adventitious adsorbed carbon monoxide demonstrates the predictive value of state-of-the-art calculation methods.
\end{abstract}




\section{Introduction}

Because defects can dominate diffusive and chemical processes on surfaces, and particularly because they are attractive to low valence species, surface impurities, whether adventitious or deposited purposely, can have an important, even a dominant effect on surface morphology and its time-evolution. With this in mind, we have explored how surface species as common as $\mathrm{H}$ and $\mathrm{O}$ modify basic surface diffusion processes on transition metal surfaces. 'The hope was, and is that the results of such studies will yield paradigms of "surfactant" behavior.

Our theoretical effort ${ }^{1}$ was motivated by the dramatic results of Esch, et al.'s Scanning Tunneling Microscopy (STM) study ${ }^{2}$ of Pt epitaxy on $\operatorname{Pt}(111)$. It showed that between 300 and $400 \mathrm{~K}, \mathrm{Pt}$-deposition on a clean surface produces 3-dimensional, pyramidal islands. But if the surface is O-precovered, then growth is virtually ideal layer-by-layer. According to Esch, et al., it is by assisting downward transport of Pt adatoms at island boundaries that $O$ inhibits island-nucleation on pre-existing islands, and thus eliminates pyramid formation. We undertook to confirm this notion theoretically, and to understand its mechanism, starting with a study of interlayer transport on O-free, stepped Pt(111).

Our Field Ion Microscope study of H-modified self-diffusion on the (100) faces of $\mathrm{Pt}^{3}$ and $\mathrm{Rh}^{4}$, and of $\mathrm{Rh}(311),{ }^{4}$ was aimed at learning whether hydrogen acts as a "skyhook," binding to an adsorbed atom and facilitating its diffusion..$^{5}$ Equally important, we wished to determine whether adsorbed $H$ has an effect on self-diffusion mechanism. This question is of considerable interest because on clean $\mathrm{Pt}(100)$, an 
"exchange diffusion" process dominates at low temperatures, ${ }^{6}$ whereby an adsorbed atom moves by substituting for a surface atom instead of simply hopping from site to site. In inhomogeneous systems, exchange is a low-temperature mechanism for surface alloying. It is thus important to know if this process can be controlled, i.e., turned on or off, by the addition of impurities.

\section{Accomplishments}

1) Theory of inter-layer self-diffusion on stepped Pt(111) - To confirm Esch, et al.'s idea of how preadsorbed $\mathrm{O}$ modifies epitaxy on $\mathrm{Pt}(111)_{2}^{2}$ we began by investigating $\mathrm{Pt}$ interlayer transport on O-free, stepped $\operatorname{Pt}(111)$. We performed $a b$ - initio -barrier calculations for downward diffusion of $\mathrm{Pt}$ adatoms at steps on $\mathrm{Pt}(111)$, with surprising results. ${ }^{1}$

The most important is that $E_{A}$, the barrier to self-diffusion down a (100)microfacet or A-type step is only $\sim 20 \mathrm{meV}$ bigger than $\mathrm{E}_{\mathrm{T}}$, the self-diffusion barrier on $\operatorname{Pt}(111)$. Thus, $E^{S}(A) \equiv E_{A}-E_{T}$, the so-called Schwoebel barrier ${ }^{7}$ that impedes transport down A-type steps, is small even in the absence of $O$. This result conflicts with Ref. 1's finding that pyramid edges on clean $\mathrm{Pt}(111)$ at $400 \mathrm{~K}$ are mainly A-steps, and with the contention that O-assisted interlayer transport is what promotes layer-by-layer epitaxy. Since pyramids grow when islands stack instead of dissipating onto lower terraces, they should be bounded by edges that present large, not minute Schwoebel barriers. Moreover, as long as A-steps form a substantial part of each island's boundary in epitaxy, as in Ref. 2, transport of Pt adatoms off islands will be facile without the assistance of adsorbed-O. 
A second surprising theoretical result is that $E_{B}$, the downward self-diffusion barrier across "B-type" or (111)-microfacet steps, is not $\sim 0.02$ but $0.35 \mathrm{eV}$ bigger than $\mathrm{E}_{\mathrm{T}}$. Thus the B-step Schwoebel barrier, $E^{\mathrm{S}}(\mathrm{B}) \equiv E_{\mathrm{B}}-\mathrm{E}_{\mathrm{T}}$, is more than an order of magnitude larger than $\mathrm{E}^{\mathrm{S}}(\mathrm{A})$. This contrast on $\mathrm{Pt}(111)$, though finally not so mysterious, is quite unexpected. The only previous ab initio study of self-diffusion on a stepped, close-packed metal surface, $\mathrm{Al}(111)$, yielded a much weaker anisotropy. ${ }^{8}$

Having computed and interpreted barriers to downward diffusion of $\mathrm{Pt}$ adatoms at steps on $\mathrm{Pt}(111)$, compared to experiment and to other theoretical work. Concerning theoretical attempts to account for the epitaxial-growth morphology of $\operatorname{Pt}(111)$ based on semi-empirica ${ }^{9 \mathrm{a}}$ or on data-fit ${ }^{\mathrm{gb}}$ energetics, comparison with our $a b$ initio energy barriers shows that this is an unlikely route to lasting, transferable interpretation. Despite some coincidences in barrier and site-occupation energies, the semi-empirical results bear no systematic resemblance to those of the $a b$ initio calculations. ${ }^{1}$

The surprisingly small Schwoebel barrier for A-steps is the most important kinetic parameter to emerge from the $a b$ initio results. Its smallness is hard to reconcile with the suggestion ${ }^{2}$ that $O$ acts as a surfactant by assisting interlayer transport. Initially this was a matter of considerable concern. But this problem was soon resolved with the discovery that $\mathrm{CO}$ contamination at very low levels, enough, however, to saturate all steps on the growing surface, was what caused the disagreement. ${ }^{10}$ When the growth experiment was repeated at much lower levels of $\mathrm{CO}$, the island morphology changed into agreement with our predictions!

Our results show that in contrast to widely applied "semi-empirical" simulations, current first-principles structural calculations are predictive for rather complex, "real- 
world" surfaces, to the extent that they can be trusted to critique the validity of experimental results. The discovery that the real effect of the $O$ is to remove small coverages of $\mathrm{CO}$ emphasizes the importance of our initial problem: Trace amounts of relatively weakly bound background gases can play an important, and in principle controllable role in determining the morphology of growing crystalline films. ${ }^{10}$

2) How adsorbed $\mathrm{H}$ affects self-diffusion on $\mathrm{Rh}$ and Pt-Manipulating Surface Diffusion Rates and Transport Mechanisms

Our direct observations of diffusing atoms in the field ion microscope show that chemisorbed hydrogen strongly influences the rate of atom migration on the $\mathrm{Rh}(100)$, $\mathrm{Rh}(311)$ and $\mathrm{Pt}(100)$ crystal planes. The influence of hydrogen is striking-pressures in the $10^{-10}$ Torr range can change the diffusion rate by several orders of magnitude. We also found that the effect of hydrogen is coverage dependent in all cases indicating that hydrogen can be used as a variable-speed control for single-atom diffusion on surfaces. Even more intriguing is the discovery that hydrogen speeds up the diffusion process on $\mathrm{Rh}(100)$ and $\mathrm{Rh}(311)$ where the mechanism is ordinary hopping, but slows it down on $\mathrm{Pt}(100)$ where the mechanism is concerted exchange. In the latter case, exchange displacements can be suppressed to the point where hopping displacements become energetically accessible. Thus, hydrogen can be used to tailor the mechanism as well as the rate of diffusion on certain surfaces. From the observed coverage dependence and opposite behavior for exchange and hopping displacements, we suggest that the effect of hydrogen is not a "skyhook"-type interact with the diffusing atom, but rather a phenomenon in which the hydrogen influences interactions with neighboring substrate atoms. This explanation is consistent with previous measurements of the effect of 
applied electric fields on surface diffusion. ${ }^{11}$ From a practical standpoint, this research has shown that hydrogen is a promising surfactant to control thin film growth and prevent intermixing at atomically sharp interfaces. 


\section{References}

1. P. J. Feibelman, Interlayer Self-Diffusion on Stepped Pt(111), Phys. Rev. Lett. 81, 168(1998).

2. S. Esch, M. Hohage, T. Michely and G. Comsa, Origin of oxygen induced layer-bylayer growth in homoepitaxy on $\operatorname{Pt}(111)$, Phys. Rev. Lett. 72, 518(1994).

3. G. L. Kellogg. Hydrogen inhibition of exchange diffusion on Pt(100), Phys. Rev. Lett., 79, 4417(1997).

4. G. L. Kellogg. Hydrogen promotion of surface self-diffusion on $\mathrm{Rh}(100)$ and Rh(311), Phys. Rev. B55,7206(1997).

5. R. Stumpf, H-enhanced mobility and defect formation at surfaces: $\mathrm{H}$ on $\mathrm{Be}(0001)$, Phys. Rev. B53, R4253-R4256 (1996)

6. P. J. Feibelman, Diffusion path for an $\mathrm{Al}$ adatom on $\mathrm{Al}(001)$, Phys. Rev. Lett. 65, 729(1990); G. L. Kellogg and P. J. Feibelman, Surface self-diffusion on Pt(001) by an atomic exchange mechanism, Phys. Rev. Lett. 64, 3143(1990); C. Chen and T. T. Tsong, Displacement distribution and atomic jump direction in diffusion of Ir atoms on the $\operatorname{Ir}(001)$ surface, Phys. Rev. Lett. 65, 3147(1990).

7. R. L. Schwoebel and E. J. Shipsey, J. Appl. Phys. 37, 3682(1966); G. Ehrlich and F. G. Hudda, J. Chem. Phys. 44, 1039(1966).

8. R. Stumpf and M. Scheffler, a) Phys. Rev. B53, 4958(1996) and b) Phys. Rev. Lett. 72, 254(1994) report, $\mathrm{ES}(\mathrm{A})=0.08 \mathrm{eV}$ and $\mathrm{ES}(\mathrm{B})=0.06 \mathrm{eV}$, for self-diffusion on stepped $\mathrm{Al}(111)$.

9. (a) M. Villarba and H. Jonsson, Surf. Sci. 317, 15 (1994); (b) J. Jacobsen, K. W. Jacobsen, P. Stoltze and J. K. Nørskov, Phys. Rev. Lett. 74, 2295(1995).

10. Matthias Kalff, George Comsa, and Thomas Michely, How Sensitive is Epitaxial Growth to Adsorbates?, Phys. Rev. Lett. 81, 168(1998).

11. G. L. Kellogg, Electric field inhibition and promotion of exchange diffusion on Pt(100), Phys. Rev. Lett. 70, 1631 (1993). 


\section{Appendices}

P. J. Feibelman, Interlayer Self-Diffusion on Stepped Pt(111), Phys. Rev. Lett. 81, 168(1998).

G. L. Kellogg. Hydrogen inhibition of exchange diffusion on $\mathrm{Pt}(100)$, Phys. Rev. Lett., 79, 4417(1997).

G. L. Kellogg. Hydrogen promotion of surface self-diffusion on $\mathrm{Rh}(100)$ and Rh(311), Phys. Rev. B55,7206(1997). 


\title{
Inter-layer self-diffusion on stepped $\mathbf{P t}(111)$
}

\author{
Peter J. Feibelman \\ Sandia National Laboratories \\ Albuquerque, NM 87185-1413
}

Challenging our understanding of epitaxy on clean and O-precovered Pt(111), the ab-initio Schwoebel barrier calculated for downward self-diffusion across A-type steps on $P t(111)$ is only $E^{S}(A) \approx 0.02 \mathrm{eV}$. Geometric arguments explain why $E^{S}(B)$, the Schwoebel barrier at B-type steps, is more than an order of magnitude larger than $E^{S}(A)$.

The time-evolution of a surface's morphology is governed by energetic barriers to diffusion of its constituents. Modern computer power and total-energy algorithms make it possible to estimate the relevant bottlenecks accurately from first principles. This means we can now lay the groundwork for reliable simulations of materials growth, aging and failure, and expect to gain meaningful, atomic-level insight into these important processes.

Because an evolving surface is inherently imperfect, the barriers that need to be computed correspond to atomic displacements not only on terraces but also near steps, kinks, vacancies and impurities. Esch, et al.'s recent Scanning Tunneling Microscopy (STM) study ${ }^{1}$ of Pt epitaxy on $\mathrm{Pt}(111)$ provides the dramatic example on which the present work is focused: Between 300 and $400 \mathrm{~K}$, Pt-deposition on the clean surface produces 3-dimensional, pyramidal islands. But if the surface is Oprecovered, then growth is virtually ideal layer-by-layer. According to Ref. 1 , it is 
by assisting downward transport of $\mathrm{Pt}$ adatoms at island boundaries that $\mathrm{O}$ inhibits island-nucleation on pre-existing islands, and thus eliminates pyramid formation.

To confirm this notion theoretically, and to understand its mechanism, it is first necessary to study interlayer transport on O-free, stepped $\mathrm{Pt}(111)$. I have therefore performed $a b$ initio barrier calculations for downward diffusion of Pt adatoms at steps on $\mathrm{Pt}(111)$, and here report the surprising results.

The most important of these is that $\mathrm{E}_{\mathrm{A}}$, the barrier to self-diffusion down a (100)-microfacet or A-type step is only $\sim 20 \mathrm{meV}$ bigger than $\mathrm{E}_{\mathrm{T}}$, the self-diffusion barrier on $\mathrm{Pt}(111)$. Thus, $\mathrm{E}^{\mathrm{S}}(\mathrm{A}) \equiv \mathrm{E}_{\mathrm{A}}-\mathrm{E}_{\mathrm{T}}$, the so-called Schwoebel barrier ${ }^{2}$ that impedes transport down A-type steps, is small even in the absence of $O$.

This result conflicts with Ref. 1's finding that pyramid edges on clean Pt(111) at $400 \mathrm{~K}$ are mainly A-steps, and with the contention that O-assisted interlayer transport is what promotes layer-by-layer epitaxy. Since pyramids grow when islands stack instead of dissipating onto lower terraces, they should be bounded by edges that present large, not minute Schwoebel barriers; and as long as A-steps form a substantial part of each island's boundary in epitaxy, as in Ref. 1, transport of Pt adatoms off islands will be facile without adsorbed-O's assistance. (Happily, new experiments show that eliminating $\mathrm{CO}$-contamination eliminates the conflict. ${ }^{3}$ )

A second surprising theoretical result is that $E_{B}$, the downward self-diffusion barrier across "B-type" or (111)-microfacet steps, is not $\sim 0.02$ but $0.35 \mathrm{eV}$ bigger than $\mathrm{E}_{\mathrm{T}}$. Thus the B-step Schwoebel barrier, $E^{S}(B) \equiv E_{B}-E_{T}$, is more than an order of magnitude larger than $E^{S}(A)$. This contrast on $\mathrm{Pt}(111)$, though finally not so mysterious, is quite unexpected. The only previous $a b$ initio study of self-diffusion on a stepped, close-packed metal surface, $\mathrm{Al}(111)$, yielded a much weaker anisotropy. ${ }^{4}$

For both A- and B-type steps (as Schwoebel and Shipsey guessed 32 years 
ago! $\left.{ }^{2}\right)$, the lowest-barrier, downward-transport mechanism is concerted substitution: the upper-terrace adatom implants in the step-edge, while a step-edge atom emerges onto the lower terrace (See Figs. 1 and 2). The key fact underlying the barrier anisotropy is that along the minimum-energy path, the latter atom is guided by its lower-terrace neighbors. The result is that at a B-step the emerging atom passes through a transition configuration where it is 3-coordinated (Fig. 2b), while at an Astep, the emergent atom never has fewer than four near neighbors (Fig. 1b). This difference is presumably more important for $\mathrm{Pt}$ than for $\mathrm{Al}$ because $\mathrm{Al}$ is trivalent.

Though this is not the first theoretical effort aimed at simulating the morphology of growing $\mathrm{Pt}(111),{ }^{5}$ when it comes to diffusion across steps, it is the first based on $a b$ initio electronic structure. This distinction is more important than anticipated -- the relative magnitudes of the first-principles Schwoebel barriers differ qualitatively from the semi-empirical estimates used in growth simulations till now. ${ }^{5}$

The results reported here, based on the Local Density Approximation (LDA), ${ }^{6}$ were obtained using the efficient and accurate total-energy and moleculardynamics package, VASP (Vienna ab-initio simulation package), ${ }^{7-9}$ its corresponding ultrasoft-pseudopotential data-base, and, to account for exchange and correlation, the Ceperley-Alder potential. ${ }^{10}$ Though plane-wave calculations for $d$-electron metals typically require unwieldy basis sets, use of an ultrasoft Pt pseudopotential with a 14 Ry basis-cutoff assures absolute convergence of total energies to 10 $\mathrm{meV}$. To accelerate electronic relaxation, I use the Fermi-level smearing approach of Methfessel and Paxton, ${ }^{11}$ with a Gaussian width of $0.2 \mathrm{eV}$. I optimize geometries till the forces on all unconstrained atoms are smaller than $0.03 \mathrm{eV} / \AA$.

To obtain baseline diffusion energetics for $\mathrm{Pt}$ on $\mathrm{Pt}(111)$, I relax 1/12 ML Pt on a 6-layer Pt(111) slab in a $3 \times 2 \sqrt{3}$ supercell, placing the Pt adatoms first in $f c c$, 
then in $h c p$ hollows, and finally finding the barrier site between them. In these calculations I fix the lower three layers of the film in the bulk, LDA atomic arrangement (LDA lattice parameter $=3.911 \AA$ ) and allow the remaining atoms to relax. To determine an adequate Surface Brillouin Zone (SBZ) sample, I perform calculations using four special $\mathrm{k}$-points in the full $\mathrm{SBZ}$, then assess convergence using a sixteen$\mathbf{k}$ sample. The corrugation of the adatom potential energy on $\operatorname{Pt}(111)$ is apparently accurate to $0.01 \mathrm{eV}$ with four $\mathbf{k}$ 's.

The $\mathrm{Pt}(111)$ calculations yield a $0.29 \mathrm{eV}$ Pt diffusion barrier on $\mathrm{Pt}(111)$, in good agreement with the values 0.25 and $0.26 \mathrm{eV}$ that emerge from Field Ion ${ }^{12}$ and Scanning Tunneling Microscope ${ }^{13}$ studies. Earlier calculations, ${ }^{12,14,15}$ not based on ultrasoft pseudopotentials, produced somewhat higher values. The best converged ${ }^{15}$ yields $(0.33 \pm 0.03) \mathrm{eV}$, consistent with the present result.

The Pt adatom preference for $f c c$ sites in the present and earlier $a b$ initio calculations $^{12,14,15}$ agrees with Field Ion Microscope (FIM) data. ${ }^{12,16}$ The $f c c-h c p$ binding-energy difference is known experimentally to be $>60 \mathrm{meV} .{ }^{16}$ The present result, $0.21 \mathrm{eV}$, is in reasonable agreement with the LDA binding difference $(0.17 \pm 0.03) \mathrm{eV}$ obtained for a smaller supercell and thinner slab. ${ }^{15}$

To estimate diffusion barriers down A- and B-type steps, I compute the energetics of $3 \times 1$ arrangements of $\mathrm{Pt}$ adatoms on 20-layer-Pt(322) and 18-layer-Pt(221) slabs (see Figs. 1 and 2), in each case fixing the lower five layers in their bulk positions as I search for the configurations that correspond to adatom diffusion barriers. Based on the Pt(111) results discussed above, and spot checks for the vicinal surfaces using $36 \mathrm{k}$ 's, I sample the (3x1)-slabs' SBZ's with sixteen k-vectors.

Key constraints on $\mathrm{Pt}$ diffusion across steps on $\mathrm{Pt}(111)$, first discussed by Villarba and Jonsson (VJ), ${ }^{6 a}$ apply equally in their semi-empirical- and the present $a b$ - 
initio-description of the process. The first constraint is imposed by the high energy of 2-coordinated $\mathrm{Pt}$ atoms. The result is that at both $\mathrm{A}$ - and B-steps, Pt-hopping over the step edge is energetically unfavorable compared to concerted substitutional diffusion (CSD). The present calculations imply that Pt-hopping over an A-type step on $\mathrm{Pt}(111)$ is $0.22 \mathrm{eV}$ more costly than CSD, and at a B-step, $0.16 \mathrm{eV}$ more costly.

In CSD down steps, the key constraint is that the low energy path for $E$, the emerging step-edge atom, is between not over its lower-terrace neighbors. This fact, as noted by both $\mathrm{VJ}^{5 \mathrm{a}}$ and $\mathrm{SS},{ }^{4 \mathrm{a}}$ means that at an A-step, atom $\boldsymbol{E}$ initially prefers not to emerge along a step normal (see Fig. 1b). Instead, it moves more directly toward its final destination (site $\beta$ in Fig. 1b), passing through a 4-coordinated configuration, at worst. At a B-step, the same constraint means that $E$ does initially displace along a normal to the edge, and must pass through a 3-coordinated site (see Fig. 2b).

Finding the A-step barrier on Pt(322): To locate the transition geometry for CSD down an A-step, it suffices to search a grid encompassing plausible barrier positions, $\left(\mathrm{x}_{E}, \mathrm{y}_{E}\right)$, of the emerging step-edge atom ( $E$, in Figs. 1$)$, where the Carte-

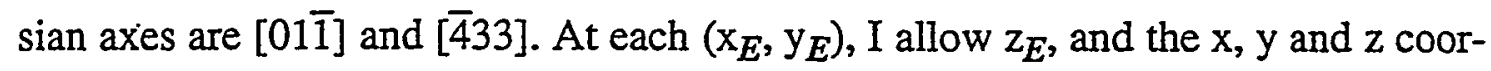
dinates of all the rest of the atoms in the $3 \times 1$ cell $^{17}$ to optimize, then compute the $\mathrm{x}$ and y-components of $\mathrm{F}$, the force on $E$. The barrier, or saddle-point, is where $\mathrm{F}$ vanishes while the determinant, $\mathrm{dF}_{\mathrm{x}} / \mathrm{dx}_{E} \mathrm{dF}_{\mathrm{y}} / \mathrm{dy}_{E}-\mathrm{dF}_{\mathrm{x}} / \mathrm{dy}_{E} \mathrm{dF}_{\mathrm{y}} / \mathrm{dx}_{E}<0$.

The result, illustrated in Fig. 1b, is that the A-step, downward CSD-barrier is only $0.31 \mathrm{eV}$, just $20 \mathrm{meV}$ larger than the barrier to Pt diffusion by hopping on the flat (111) terrace. Thus, only a small Schwoebel barrier (close to the accuracy limit of the present calculations) hinders downward transport of upper-terrace Pt adatoms. This result, which demands that we reconsider why Pt grows 3-dimensionally on $\mathrm{Pt}(111)$ between 300 and $400 \mathrm{~K}$, is not entirely unexpected. It is in fact quite sim- 
ilar to Ref. 4's result for CSD on A-stepped Al.

Incidentally, the A-step CSD-barrier obtained semi-empirically by VJ,, 0.30 $\mathrm{eV}$, is very close to that obtained here. VJ's Schwoebel barrier is nonetheless much higher than $0.02 \mathrm{eV}$ because their terrace-diffusion barrier is unphysically low.

By way of interpretation, VJ contend that the A-step CSD-barrier is "big", because to avoid step-bottom atom $B$, emerging atom $E$ must pass close to edge neighbor, $N$. In fact, it is precisely because $\overline{E N}$ is relatively small that $E$ is effectively 4-coordinated in the barrier geometry. According to the $a b$ initio calculations, distances $\overline{E N}$ and $\overline{E B}$ both equal $\sim 2.52 \AA$, about $9 \%$ less than the bulk nearestneighbor separation, $2.77 \AA$. At the same time, $E$ is $2.61 \AA$ from its other lower terrace neighbor (blocked from view in Fig. $1 \mathrm{~b}$ ), and $\sim 2.44 \AA$ from $A$. Relatively small values of $\overline{E N}, \overline{E B}$ and $\overline{E A}$ are favored for two reasons. The first is the usual "bondorder bond length correlation." 18 The second is that Pt surfaces are typically under tensile stress, which is relieved when low symmetry allows bonds to shorten. ${ }^{19}$

Finding the B-step barrier on $\mathbf{P t}(221)$ : An extensive search for the downward-CSD barrier-geometry at a B-step again confirms that the emergent step-edge atom is initially guided by its lower-terrace neighbors. A consequence, cf. Fig. 2b, is symmetry of the transition geometry under reflection in a $y-z$ plane (where $x-, y-$ and $z$-coordinates lie along $[\overline{1} 10],[\overline{11} 4]$ and $[221])$. Although this symmetry suggests that to find the barrier one can simply drag the y-coordinate of atom $E \mid$ in the $y$-direction, looking for a maximum in the energy, this simple procedure fails. Instead of a smooth curve with a maximum where its slope vanishes, one finds that $F_{y}$ vs. $y$ has two concave-upwards segments meeting at a cusp. The reason is that on either side of the cusp, other coordinates of the many-atom system relax differently.

Again, therefore, a two-dimensional grid search is required. In the present 
case, I fix the y-coordinates of atom $E$, and of its immediate step-edge neighbors, $N$. The upshot is a transition geometry (see Fig. $2 \mathrm{~b}$ ) in which adatom $\boldsymbol{A}$ has moved to a position near the $h c p$ hollow behind $E$. At the same time, edge neighbors, $N$, also trailing $E$, have fallen somewhat behind it.

The calculated barrier is $0.64 \mathrm{eV}$. Thus, downward transport at a B-type step requires $0.35 \mathrm{eV}$ more than diffusion on the flat $\mathrm{Pt}(111)$ terrace, and at low-T, CSD is much less probable at a B-type than at an A-type step.

Why is downward diffusion so much more costly at a B-type as against an Atype step? Neighbor-counting provides a compelling clue. At the B-step saddle, illustrated in Fig. 2b, emerging atom $E$ has only three near neighbors, adatom $A$ and bottom atoms $B$. That is, while $\overline{E A}$ and $\overline{E B}$ equal 2.44 and $2.55 \AA, E$ 's next-nearest neighbors, atoms $N$, are $2.80 \AA$ distant. In the A-step transition geometry, as detailed above, the emergent atom has four near neighbors.

Another difference between diffusion processes at A- and B-type steps, also favoring A-type, is worth bearing in mind: As illustrated in Figs. 1 and 2, in both cases, the adatom moves along the step from an initial edge-adjacent $f c c$-hollow to a position near an $h c p$-site in the transition geometry. But as the energies shown in Figs. 1a and 2a imply, adatom displacement along the A-step edge is more facile than along a B-step. The energy surface is more corrugated along the B-step because the advantages of being in an $f c c$ hollow, and of being coordinated to (and thus passivating) two step-edge atoms, are in phase. Adjacent to an A-step they are not. There, an adatom can either reside in an $f c c$ hollow and have one step-edge neighbor, as in Fig. 1a, or it can occupy an $h c p$ site have two. This is why the fcc$h c p$ binding difference is only $0.03 \mathrm{eV}$ along an $\mathrm{A}$-step, but $0.19 \mathrm{eV}$ at a B step, and presumably why the barriers to move along A- and B-steps are 0.18 vs. $0.30 \mathrm{eV}$. 
Having computed and interpreted downward CSD-barriers at steps on $\mathrm{Pt}(111)$, I now wish to compare to experiment and to other theoretical work. Based on FIM observations, Ref. 16 reports that Pt atoms adsorbed at the tops of island edges on Pt(111) only incorporate into the edges somewhat above $130 \mathrm{~K}$. Whether this observation is consistent with a low barrier to CSD at A-steps requires detailed structural information not provided in Ref. 16, e.g., whether the adatom incorporation occurs at kinks, comers or at A-type steps.

The main point of Ref. 16 is that $\mathrm{Pt}$ adatoms on islands avoid a zone 2-3 nearest-neighbor spacings wide, starting one spacing inside the island boundary. The (5atomic-row wide) terraces on the $\mathrm{Pt}(322)$ slab studied here are too narrow to make a detailed comparison with this observation. Nonetheless (see Fig. 1a) adatom binding energies on $\mathrm{Pt}(322)$ do not monotonically increase as one moves onto the upper terrace from the step edge, but rather, show a maximum.

Concerning attempts to account for the epitaxial-growth morphology of $\mathrm{Pt}(111)$ based on semi-empirical ${ }^{5 \mathrm{a}}$ or on data-fit ${ }^{5 \mathrm{~b}}$ energetics, Table 1 shows that this is an unlikely route to lasting, transferable interpretation. Despite some coincidences in barrier and site-occupation energies, the semi-empirical results bear no systematic resemblance to those of the ab initio calculations.

The surprisingly small Schwoebel barrier for A-steps is the most important kinetic parameter to emerge from the $a b$ initio results. Its smallness is hard to reconcile with the suggestion ${ }^{1}$ that $O$ acts as a surfactant by assisting interlayer transport. Learning from a contradiction of this nature is virtually impossible if one starts from a theoretical method of questionable accuracy, and completely impossible if one works backward from experiment. New studies, ${ }^{3}$ attributing Ref. 1's growthmorphology transition to CO-contamination, underline this caution. 
Acknowledgments - I thank R. Stumpf and T. Michely for numerous discussions, and the latter and M. Kalff for access to their unpublished results. VASP ${ }^{7-9}$ was developed at the Institut für Theoretische Physik of the Technische Universität Wien. This work was supported by the U. S. Department of Energy under Contract No. DE-AC04-94AL85000. Sandia is a multi-program laboratory operated by Sandia Corporation, a Lockheed-Martin Company, for the U. S. Department of Energy. 


\section{REFERENCES -}

1. S. Esch, M. Hohage, T. Michely and G. Comsa, Phys. Rev. Lett. 72, 518(1994).

2. R. L. Schwoebel and E. J. Shipsey, J. Appl. Phys. 37, 3682(1966); G. Ehrlich and F. G. Hudda, J. Chem. Phys. 44, 1039(1966).

3. M. Kalff, G. Comsa and T. Michely (unpublished) show that background $C O$, below its desorption temperature of $400-500 \mathrm{~K}$, is what stabilized the A-stepbounded islands and pyramids of Ref. 1, and infer that $\mathrm{O}$ simply removed the $\mathrm{CO}$. 4. R. Stumpf and M. Scheffler, a) Phys. Rev. B53, 4958(1996) and b) Phys. Rev. Lett. 72, 254(1994) report, $E^{S}(A)=0.08 \mathrm{eV}$ and $E^{S}(B)=0.06 \mathrm{eV}$, for self-diffusion on stepped $\mathrm{Al}(111)$.

5. See, e.g., a) M. Villarba and H. Jonsson, Surf. Sci. 317, 15 (1994) and b) J. Jacobsen, K. W. Jacobsen, P. Stoltze and J. K. Nørskov, Phys. Rev. Lett. 74, 2295(1995). 6. See The Theory of the Inhomogeneous Electron Gas, edited by S. Lundqvist and N. H. March (Plenum Press, New York, 1983); also, W. E. Pickett, Comp.|Phys. Rep. 9, 115(1989).

7. G. Kresse and J. Hafner, Phys. Rev. B47, 558 (1993); ibid. B49, 14251 (1994).

8. G. Kresse and J. Furthmüller, Comput. Mat. Sci. 6, 15 (1996).

9. G. Kresse and J. Furthmüller, Phys. Rev. B54, $11169^{\circ}$ (1996).

10. D. M. Ceperley and B. J. Alder, Phys. Rev. Lett. 45, 566(1980), as parameterized by J. Perdew and A. Zunger, Phys. Rev. B23, 5048(1981).

11. M. Methfessel and A. T. Paxton, Phys. Rev. B40, 3616(1989).

12. P. J. Feibelman, J. S. Nelson and G. L. Kellogg, Phys. Rev. B49, 10548(1994).

13. M. Bott; M. Hohage, M. Morgenstern, T. Michely and G. Comsa, Phys. Rev. Lett. 76, 1304(1996).

14. J. J. Mortensen, B. Hammer, O. H. Nielsen, K. W. Jacobsen and J. K. Nørskov, 
Springer Series on Solid State Physics, ed. by A. Ojiki, 121, 173(1996).

15. G. Boisvert, L. J. Lewis and M. Scheffler, Phys. Rev. B57, 1881(1998).

16. A. Gölzhäuser and G. Ehrlich, Phys. Rev. Lett. 77, 1334(1996).

17. Except the five bottom layers, as noted above.

18. L. Pauling, The Nature of the Chemical Bond (Comell Univ. Press, Ithaca, NY, 1960), 3rd ed..

19. R. J. Needs, M. J. Godfrey and M. Mansfield, Surf. Sci. 242, 215(1991). 


\section{Figure Captions -}

1. a) Initial and b) downward-diffusion barrier geometries for the A-type step of $\mathrm{Pt}(322)$. The adatom, the emergent edge-atom (destined for site $\beta$ ), and its nearest neighbors in the step edge and at the step bottom are labelled $A, E, N$, and $B$. Calculated energies (in $\mathrm{eV}$ ) shown at various adatom sites, are defined relative to the configuration illustrated in panel a).

2. a) Initial b) downward-diffusion barrier geometries for the B-type step of Pt(221). The adatom, the emergent edge-atom, and its nearest neighbors in the step edge and at the step bottom are labelled $A, E, N$, and $B$. Energies (in $\mathrm{eV}$ ) shown at various adatom sites, are defined relative to the configuration illustrated in panel a). 


\section{Table Caption -}

1. A $b$ initio vs. semi-empirical activation barriers for self-diffusion on terraces, and down A- and B-type steps on Pt(111). VASP, EAM and EMT results refer to the . present work, the Embedded Atom Method and Effective Medium Theory. 
Table 1:

\begin{tabular}{|c|c|c|c|}
\hline barrier & VASP & EAM $^{\mathrm{a}}$ & \multicolumn{1}{|c|}{ EMT $^{\mathrm{b}}$} \\
\hline \hline hop on (111)-terrace & $0.29 \mathrm{eV}$ & $0.08 \mathrm{eV}$ & $0.16 \mathrm{eV}$ \\
\hline exchange down A-step & $0.31 \mathrm{eV}$ & $0.30 \mathrm{eV}$ & "not low" \\
\hline exchange down B-step & $0.64 \mathrm{eV}$ & $0.18 \mathrm{eV}$ & $0.37 \mathrm{eV}$ \\
\hline hop down A-step & $0.53 \mathrm{eV}$ & not given & $0.41 \mathrm{eV}$ \\
\hline hop down B-step & $0.80 \mathrm{eV}$ & not given & not given \\
\hline
\end{tabular}

${ }^{a}$ Ref. 5a

${ }^{b}$ Ref. 5b 
Fig. 1a

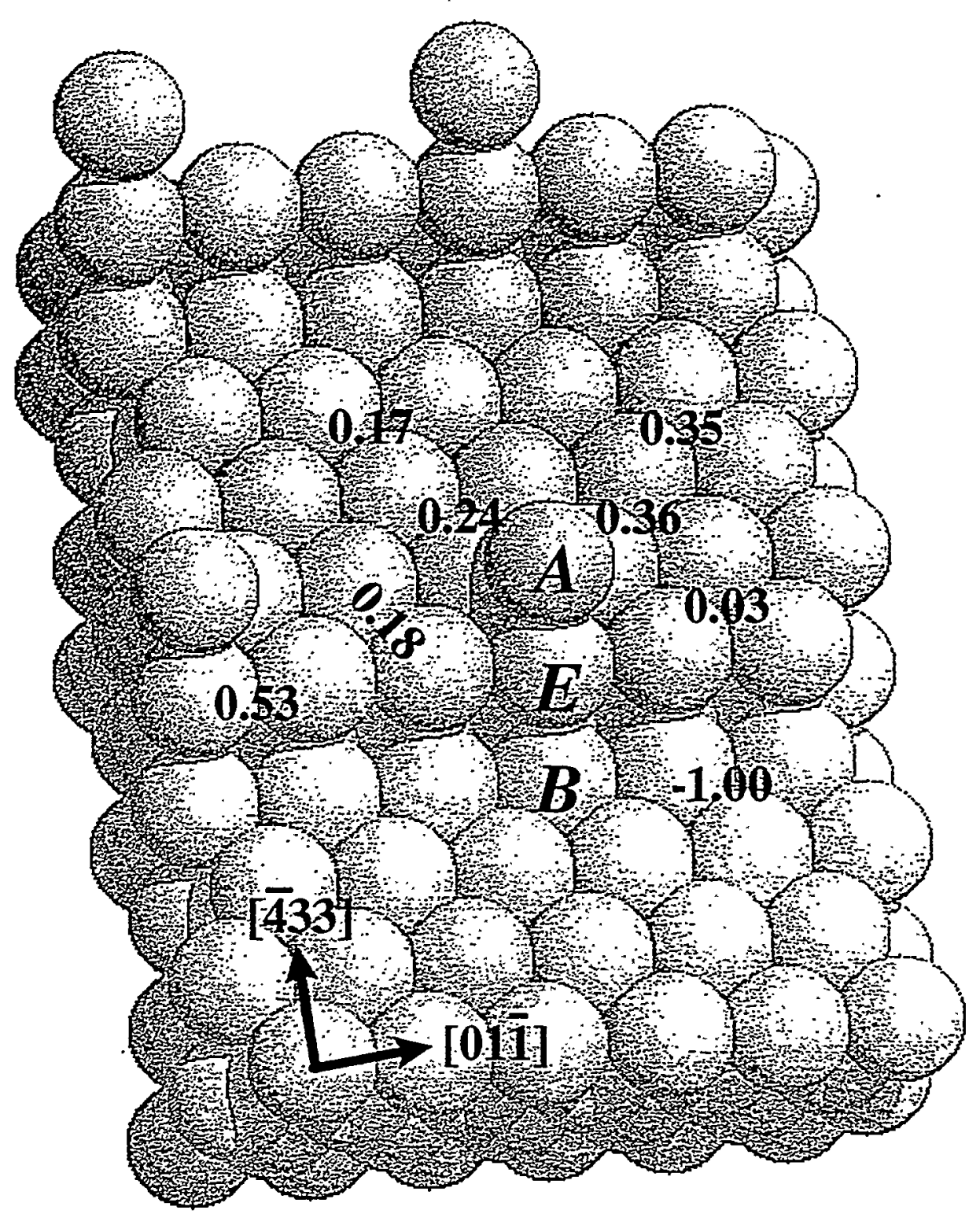


Fig. 1b

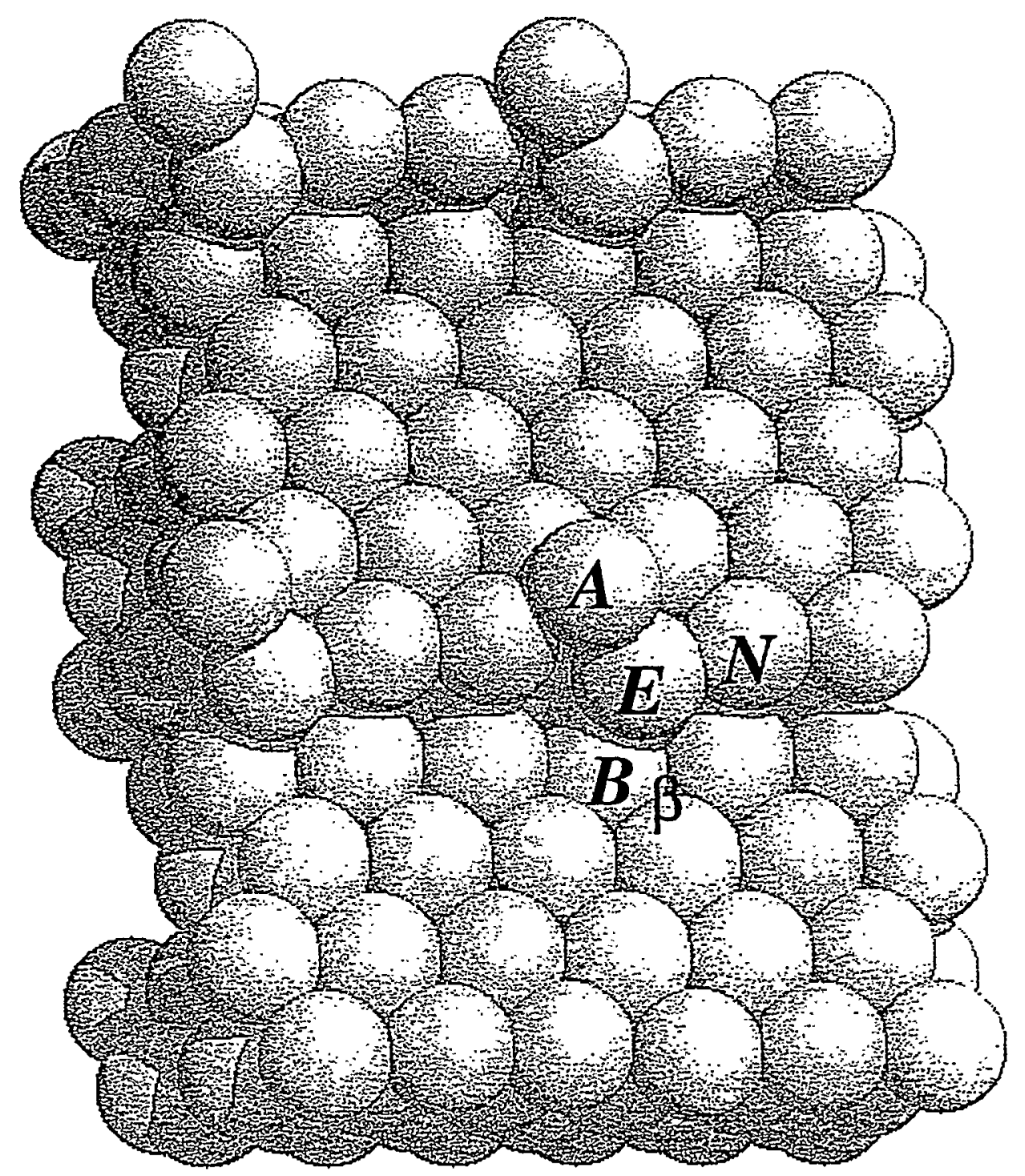


Fig. 2a

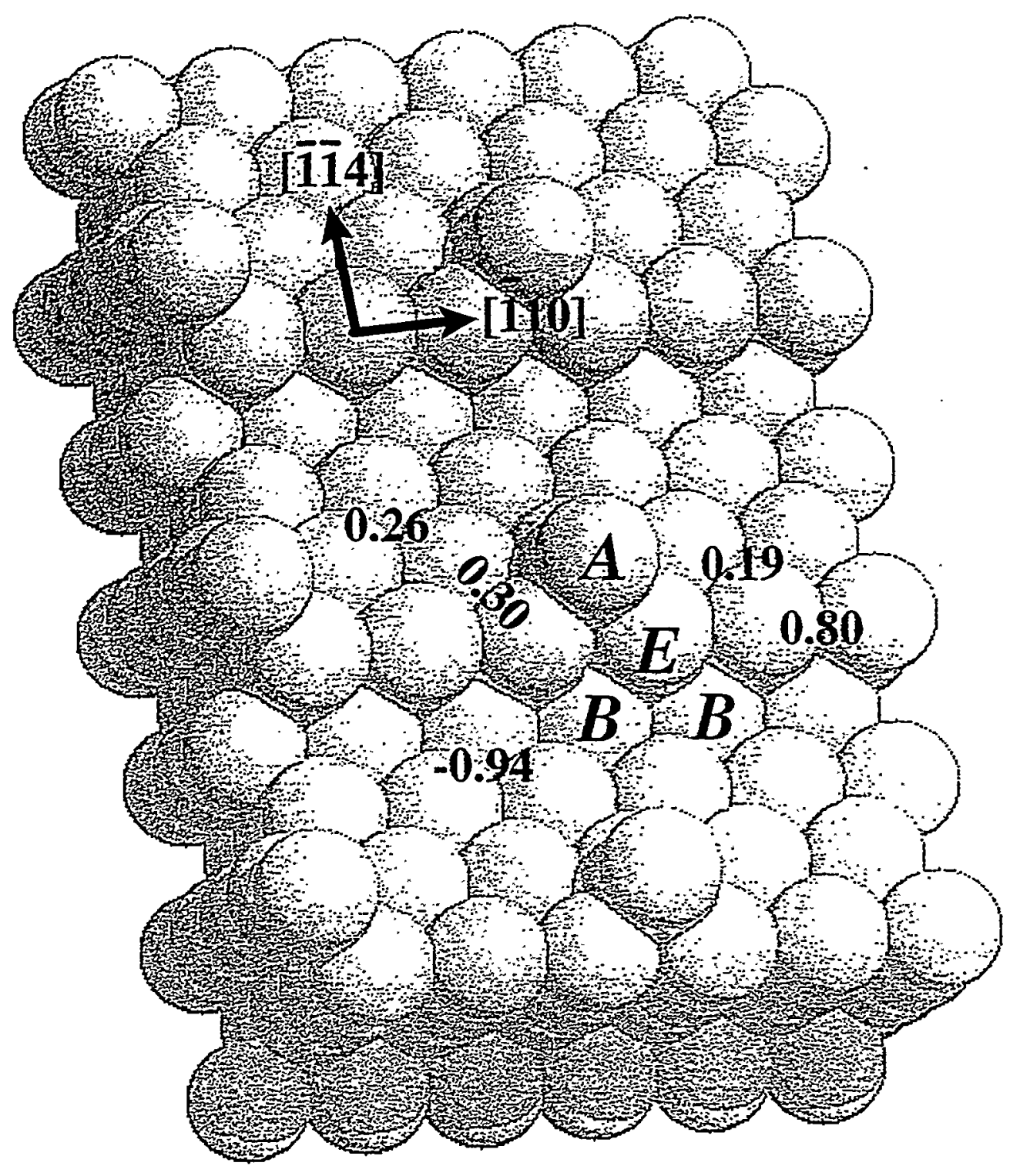


Fig. 2b

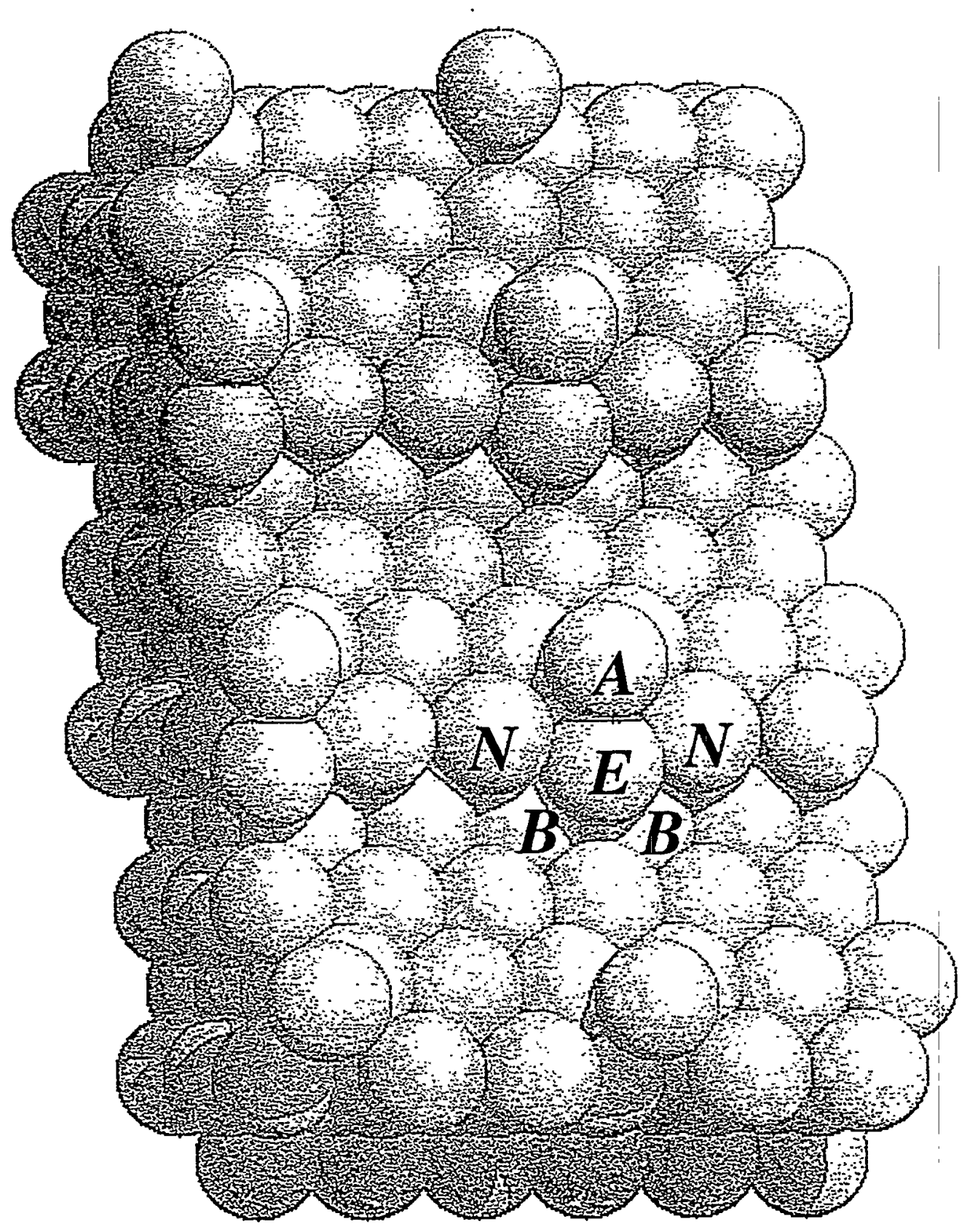




\title{
Hydrogen Inhibition of Exchange Diffusion on Pt(100)
}

\author{
G.L. Kellogg* \\ Sandia National Laboratories, Albuquerque, New Mexico 87185-1413
}

(Received 9 June 1997)

\begin{abstract}
Field ion microscope observations show that the diffusion rate of $\mathrm{Pt}$ atoms on $\mathrm{Pt}(100)$ is significantly reduced when the surface is exposed to hydrogen. A hydrogen partial pressure of $1 \times 10^{-10}$ Torr causes the exchange diffusion rate to decrease by $\sim 3$ orders of magnitude over 23 hours. Higher pressures suppress exchange displacements to the point where hopping displacements become energetically accessible. The ability to influence both the displacement rate and the transpor mechanism indicates that hydrogen may be used to control thin-film growth at the atomic level.
\end{abstract} [S0031-9007(97)046346]

PACS numbers: 68.35.Fx, 61.16.FK, 66.30.Fq, 68.55.-a

The migration of individual atoms across single-crystal terraces and their incorporation into the top layer of surface atoms play important roles in the growth of crystalline solids and epitaxial films. The development of methods to manipulate these fundamental processes at the atomic level offers the exciting possibility to tailor crystal and thin-film growth for specific materials applications. Indeed, past studies have shown that adsorbed atoms or molecules, often referred to as "surfactants," can be used either to change the surface morphology of growing films [1] or to suppress intermixing at the substrate-film interface [2]. Although it is often assumed that the observed changes in these studies are due to the effect of the foreign species on surface diffusion, there are a number of elementary steps involved in the overall growth process, any or all of which may be subject to modification by adsorbates. To sort out the details of the different effects, one must isolate the individual steps of the growth process and determine how a given adsorbate affects each one individually. Whether considering atom migration across terraces or atom incorporation into the surface layer, one specifically needs to know if a given adsorbate will promote or inhibit the process.

Single-atom diffusion on the (100) crystal planes of fcc metals is an excellent model system for investigation of adsorbate-mediated surface diffusion and atom incorporation processes. It has been shown that surface diffusion on these surfaces proceeds by one of two mechanisms: ordinary hopping or exchange [3]. Hopping results from the adatom being displaced over the minimum in the potential barrier between two adjacent binding sites, whereas exchange displacements involve a process in which the adatom plunges down into the surface layer and pushes a surface atom up into a diagonally opposite fourfold hollow. By studying surface diffusion on fec $(100)$ surfaces, one can derive information on how adsorbates affect both the rate of surface diffusion as well as the energy barrier for incorporation of adatoms into the surface layer.

Hydrogen is a common adsorbate used in a variety of surface science studies and appears to be a good candidate for the modification of epitaxial growth on both metal [1(a)] and semiconductor surfaces [4]. In this sudy the field ion microscope (FIM) is used to investigate how hydrogen influences the diffusion of single $\mathrm{Pt}$ atoms on $\mathrm{Pt}(100)$. Past studies show that self-diffusion on $\mathrm{Pt}(100)$ proceeds exclusively by the exchange mechanism at temperatures from $160-230 \mathrm{~K}$ [3]. The purpose of this investigation is to determine if hydrogen enhances or inhibits diffusion by exchange displacements and whether or not it can change the preferred mechanism from exchange to hopping.

The experimental procedures used in FIM investigations of single-atom surface diffusion are described in detail in several recent review articles [5] and are not repeated here. In the present experiments, a single $\mathrm{Pt}$ adatom is generated by field evaporation of the topmost (100) plane. The field evaporation process is quickly terminated when a single atom remains on the surface. A sequence of diffusion intervals is carried out to establish the mean-square displacement of the adatom in the absence of hydrogen. Without removing the imaging gas (neon at $2 \times 10^{-4}$ Torr), hydrogen is leaked into the system to a preset partial pressure. The leak valve is calibrated in control experiments (without $\mathrm{Ne}$ ) to determine the partial pressure of added hydrogen. Subsequent measurements are made with the mixture of neon and hydrogen as the imaging gas. The partial pressure of hydrogen in this study is in the low $10^{-10}$ to $10^{-9}$ Torr range. At lower partial pressures, hydrogen has a negligible effect on the diffusing adatoms. At hydrogen partial pressures above $\sim 10^{-8}$ Torr, $\mathrm{Pt}$ adatoms field desorb from the surface during field-ion imaging [6]. The background pressure of the system without hydrogen or neon is $2 \times 10^{-11}$ Torr.

A series of field-ion micrographs indicating the effect of hydrogen on self-diffusion on $\operatorname{Pt}(100)$ is shown in Fig. 1. Figure 1(a) shows a single $\mathrm{Pt}$ adatom on $\mathrm{Pt}(100)$ imaged in pure Ne at $77 \mathrm{~K}$. Figures 1(b)-1(d) show the same adatom after 30 -sec heating intervals at $200 \mathrm{~K}$ in pure Ne. Displacements of the adatom across the surface are obvious. The mean-square displacement at this temperature is several hundred $\AA^{2}$. Figures $1(\mathrm{e})-1(\mathrm{f})$ show the 


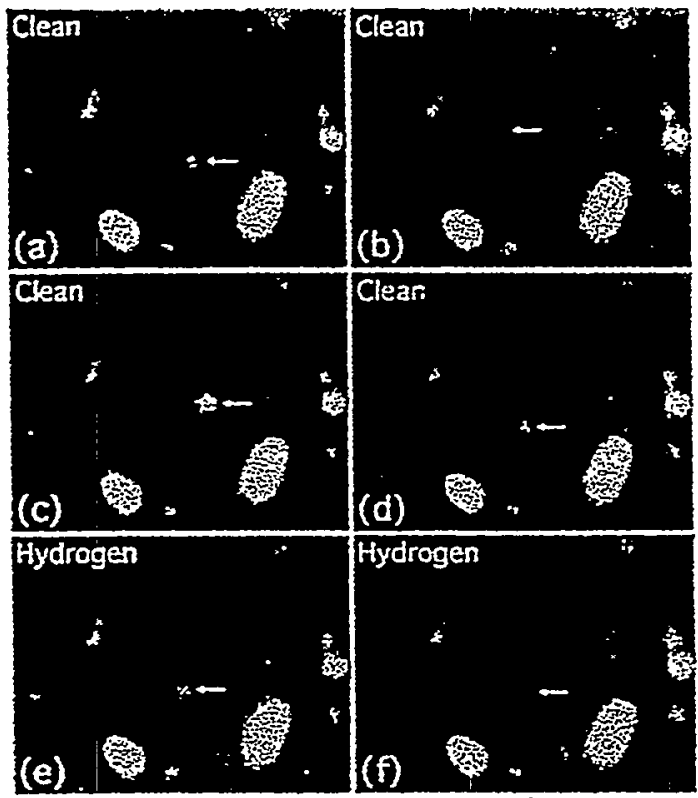

FIG. 1. Field-ion microscope images showing the diffusion of an individual $\mathrm{Pt}$ adatom on Pt(100). Between each photograph the sample was warmed from its base temperature of 77 to $200 \mathrm{~K}$. The introduction of $\mathrm{H}_{2}$ between (d) and (e) stops the migration of the adatom.

same adatom after admission of hydrogen at a partial pressure of $1 \times 10^{-9}$ Torr. Measurements of the adatom's coordinates indicate that the adatom is now stationary during the heating intervals. In subsequent diffusion intervals no further motion of the adatom is detected. Thus, exposure of the surface to hydrogen at a partial pressure of $1 \times 10^{-9}$ Torr completely stops self-diffusion on $\mathrm{Pt}(100)$ at $200 \mathrm{~K}$.

Although it is not possible to determine the hydrogen coverage with the present experimental setup, we can examine trends associated with the coverage by measuring the adatom's mean-square displacement as a function of time after admission of a fixed hydrogen partial pressure and as a function of the hydrogen partial pressure itself. Figure 2 shows plots of the measured mean-square displacement as a function of time after admission of $1 \times 10^{-10}$ and $2 \times 10^{-10}$ Torr hydrogen. The diffusion intervals are $30 \mathrm{sec}$ in length at a temperature of $195 \mathrm{~K}$. The bin size for each data point is 25 diffusion intervals. It is obvious that the mean-square displacement decreases monotonically as a function of time after adding hydrogen to the system. This monotonic decrease indicates that the inhibition of self-diffusion is dependent on the hydrogen coverage. As the amount of hydrogen builds up on the surface, the diffusion rate decreases. It is also obvious from Fig. 2 that doubling the hydrogen partial pressure causes the mean-square displacement to decrease much more rapidly as a function of time. This observation is furher evidence that the inhibition due to hydrogen is coverage dependent

4418

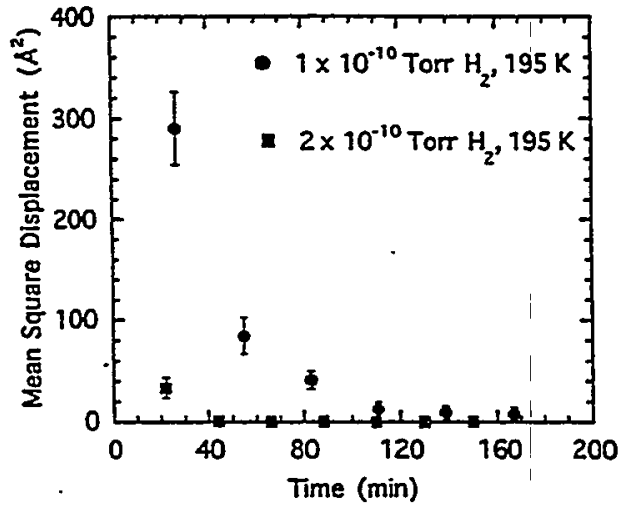

FIG. 2. The mean-square displacement of a Pt adatom diffusing on $\mathrm{Pt}(100)$ at $195 \mathrm{~K}$ in the presence of $\mathrm{H}_{2}$ decreases continuously as a function of time. The decrease is much faster in $2 \times 10^{-10}$ Tor $\mathrm{H}_{2}$ (squares) than $1 \times 10^{-10}$ Torr $\mathrm{H}_{2}$ (circles).

Figure 3 shows a similar plot for the case in which the hydrogen partial pressure is $3 \times 10^{-10}$ Torr. Here, the mean-square displacement immediately falls to zero, i.e., the diffusion completely stops upon exposure to hydrogen. This plot also shows the result of increasing the temperature to $230 \mathrm{~K}$ following $90 \mathrm{~min}$ of heating cycles at $195 \mathrm{~K}$ in $3 \times 10^{-10}$ Torr H2. The adatom, which is completely immobile for $90 \mathrm{~min}$ at $195 \mathrm{~K}$, makes significant displacements at $230 \mathrm{~K}$. The measured mean square displacement upon increasing the temperature is $\sim 100 \AA^{2}$. Note that after increasing the temperature, the mean-square displacement does not stay constant as a function of time, but gradually decreases over the next $2 \mathrm{~h}$. This implies that even though the atom is immobile during the first $90 \mathrm{~min}$ at $195 \mathrm{~K}$, the surface is not totally saturated with hydrogen. In a background of $3 \times 10^{-10} \mathrm{Tor}^{\mathrm{H}} \mathrm{H}_{2}$, the surface coverage increases continuously over a period of several hours.

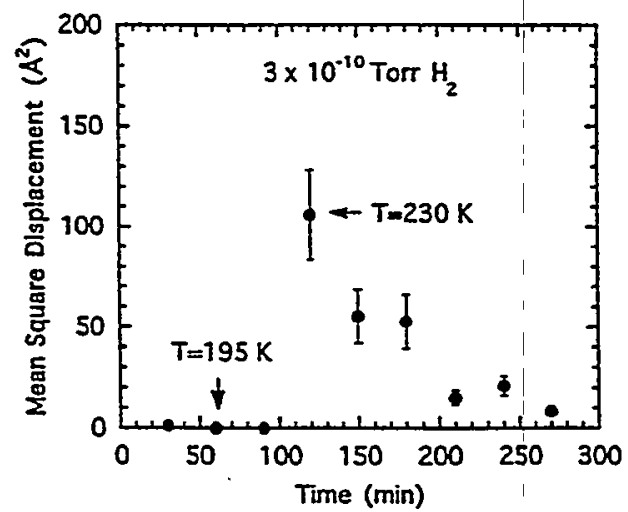

FIG. 3. In $3 \times 10^{-10}$ Torr $\mathrm{H}_{2}$ the mean-square displacement at $195 \mathrm{~K}$ falls to zero immediately. Increasing the temperature to $230 \mathrm{~K}$ after $90 \mathrm{~min}$ of $\mathrm{H}_{2}$ exposure causes the adatom to move. The mean-square displacement the higher temperarure also falls off as a function of time. 
Having established a pronounced effect of hydrogen on the diffusion rate, we next address its effect on the diffusion mechanism. As shown in previous studies, one can distinguish between exchange displacements and hopping displacements on fcc (100) surfaces by examination of the map of sites the adatom visits as it migrates across the surface [3]: Figure 4(a) shows the map for a Pt adatom diffusing on $\mathrm{Pt}(100)$ at $175 \mathrm{~K}$ in the absence of hydrogen. As found in previous studies [3], the square pattern has sides parallel to $\langle 001\rangle$ [i.e., the map is $c(2 \times 2)$ ] implying diffusion by exchange. Figures $4(\mathrm{~b})-4(\mathrm{~d})$ show site visitation maps for higher partial pressures of hydrogen and higher temperatures. At temperatures of 195 and $230 \mathrm{~K}$ the maps remain $c(2 \times 2)$ indicating that the diffusion mechanism at these temperatures is still exclusively exchange [Figs. 4(b) and 4(c)]. The hydrogen background pressure in these experiments was $1 \times 10^{-10}$ Torr and $3 \times 10^{-10}$ Torr, respectively. Under these conditions, the hydrogen strongly affects the diffusion rate, but not the mechanism. At $250 \mathrm{~K}$ and approximately $1 \times 10^{-9}$ Tor $\mathrm{H}_{2}$, the map becomes $(1 \times 1)$ [Fig. 4(d)]. This is direct experimental evidence that hopping displacements become accessible for self-diffusion on $\mathrm{Pt}(100)$ at temperatures between $230-250 \mathrm{~K}$. At these temperatures, it is difficult to collect statistical samples equivalent to those obtained at lower temperatures because the adatom typically migrates off the plane edge within a few diffusion cycles.

It should be noted that diffusion at $250 \mathrm{~K}$ in the absence of hydrogen cannot be examined in the FIM because

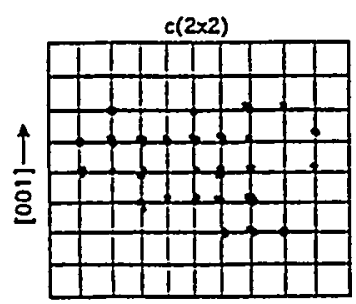

(a)

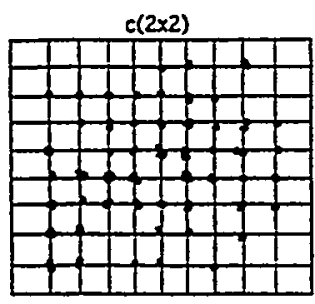

(c) $T-230 \mathrm{~K}$

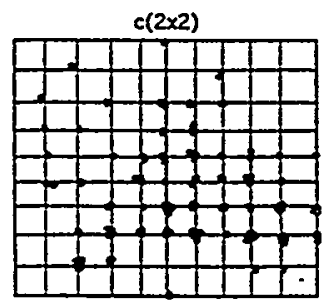

(b)

$T=195 \mathrm{~K}$

(ixi)

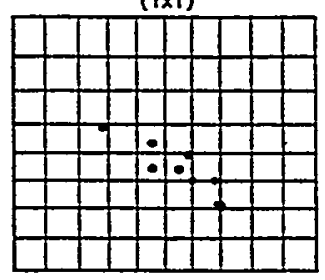

(d) $\quad T-250 \mathrm{~K}$
FIG. 4. Site visitation maps for $\mathrm{Pt}$ on $\mathrm{Pt}(100)$ for different temperarures and $\mathrm{H}_{2}$ parrial pressures. (a)-(c) Maps with $c(2 \times 2)$ periodicity indicate that the mechanism of displacement is exclusively exchanged for temperatures of (a) $175 \mathrm{~K}$; (b) $195 \mathrm{~K}$; and (c) $230 \mathrm{~K}$. (d) $\mathrm{A}(1 \times 1)$ map for diffusion at $250 \mathrm{~K}$ indicates that hopping displacements take place. The $\mathrm{H}_{2}$ partial pressures are (a) 0 ; (b) $\mathrm{I} \times 10^{-10} \mathrm{Torr}$ (c) $3 \times 10^{-10}$ Torr, and (d) $1 \times 10^{-9}$ Torr. exchange diffusion at $250 \mathrm{~K}$ is so fast that the adatom migrates off the crystal plane in a single diffusion interval. Thus, the effect of hydrogen is to inhibit exchange displacements to the point where hopping displacements can be observed. From the onset temperature for hopping displacements and the assumption of the conventionally accepted prefactor, the activation energy for hopping displacements is estimated to be $0.63-0.66 \mathrm{eV}$. This activation energy is nearly $0.2 \mathrm{eV}$ higher than the measured activation energy for exchange displacements on $\mathrm{Pt}(100)$ and is consistent with estimates based on a previous study in which exchange displacements were inhibited by a high electric field [7].

The results reported here lead to three important conclusions concerning hydrogen's effect on $\mathrm{Pt}(100)$ self-diffusion: (1) small amounts of adsorbed hydrogen decrease the rate of exchange displacements on $\mathrm{Pt}(100)$; (2) the effect of hydrogen is coverage dependent; and (3) exchange displacements can be suppressed to the point where the rates of hopping and exchange displacements become comparable. The first observation is significant in that the effect of hydrogen on Pt(100) self-diffusion is opposite to that found for self-diffusion on $\mathrm{Rh}(100)$ and $\mathrm{Rh}$ (311) [8]. In these systems, for which the displacement mechanism is ordinary hopping, increasing coverages of hydrogen actually increase the diffusion rate. Although more systems need to be studied to establish a true correlation, the results point to the intriguing possibility that chemisorbed hydrogen, in general, promotes hopping displacements but inhibits exchange displacements. If true, this would establish hydrogen as an extremely useful and versatile tool for manipulating thin film grown at the atomic level.

Equally imporant with respect to manipulating nucleation and growth processes is the observation that the effect of hydrogen is coverage dependent over a large range of diffusion rates. The ability to adjust the diffusion rate of single atoms on terraces in a continuous manner provides an exceptional degree of control in guiding atomicscale processes to achieve a desirable growth morphology. This is especially true in light of the previous observation that the promotion of hopping displacements by hydrogen is also coverage dependent [8]. The third observation concerning the effect of hydrogen on the displacement mechanism has interesting consequences in relation to the growth of thin surface films and multilayered strucures. Typically, one wishes to grow structures with atomically sharp interfaces. However, if exchange displacements have a lower activation banier than hopping displacements, there will always be intermixing at the interface at temperanures required for growth. These results thus identify hydrogen as a promising candidate for suppression of interfacial intermixing in the growth of multilayered thin films.

Although this investigation has established that hydrogen has a strong infiuence on both the diffusion rate and the transport mechanism for $\mathrm{Pt}$ atoms on $\mathrm{Pt}(100)$, the experiments in themselves do not identify the underlying cause 
of the effect. The $P t(100)$ surface examined in this study is an unreconstructed $(1 \times 1)$ surface prepared by field evaporation at low temperatures. This is not the stable structure produced by standard sputtering and annealing techniques. As a result, there have been no temperature programmed desorption or vibrational spectroscopic studies of hydrogen on the unreconstructed $\mathrm{Pt}(100)$ surface to characterize the nature of hydrogen adsorption. However, based on studies of hydrogen adsorption on other surfaces of $P t$ [9], as well as on the (100) surfaces of other fcc metals [10], it is reasonable to assume that hydrogen adsorbs dissociatively on $\operatorname{Pt}(100)$ with a relatively high sticking coefficient. This implies that the observed inhibition of self-diffusion is due to chemisorbed atomic hydrogen.

How might chemisorbed hydrogen inhibit exchange displacements? In general, exchange displacements are energetically favorable when the energy gain due to the adatom's higher coordination at the saddle point of the transition compensates for the energy cost in creating a surface vacancy to achieve this configuration. Inhibition can be achieved either by increasing the height of the barrier at the saddle configuration or by increasing the vacancy formation energy. It has been suggested that on clean fcc (100) surfaces, lateral relaxations of neighboring surface atoms due to the presence of the adatom reduce the vacancy creation energy and promote exchange displacements [11]. In fact, inhibition of exchange displacements due to an externally applied electric field was explained in terms of a model in which a field-induced charge transfer lifted the atom off the surface and reduced the surface atom relaxations [7]. It is conceivable that a hydrogen atom, chemisorbed on top of a Pt adatom, could have the same effect. Charge transfer from the adatom to the hydrogen could reduce the surface relaxations and inhibit exchange displacements. However, the observed coverage dependence would suggest that the chemisorption would necessarily be transitory in this type of process; i.e., the hydrogen leaves the adatom after every diffusion event.

More likely is the scenario in which mobile hydrogen atoms visit sites next to the $\mathrm{Pt}$ adatom and increase the diffusion barrier by increasing the energy required to bring a surface atom up from the substrate. At low coverages the probability of a hydrogen being in a neighboring site is low. As the coverage increases, so does the probability of a hydrogen occupying a neighboring site. The prevention of an exchange displacement by a hydrogen atom in a neighboring site is consistent with the observed coverage dependence. On a macroscopic scale one could view this phenomenon as an increase in surface stress brought about by the adsorption of hydrogen. Recent calculations show a convincing correlation between surface stress and the propensity for exchange displacements on fcc (100) surfaces [12]. Clearly, more modeling of this sort is needed to explain the mechanism of hydrogen inhibition observed in this investigation.

It is a pleasure to acknowledge many fruifful discussions with Brian Swartzentruber throughout the course of this investigation. Sandia is a multiprogram/laboratory operated by Sandia Corporation, a Lockheed Martin Company, for the United States Department of Energy under Contract No. DE-AC04-94AL85000.

*Electronic address: glkello@sandia.gov

[1] (a) W.F. Egelhoff, Jr. and D.A. Steigerwald, J. Vac. Sci. Technol. A 7, 2167 (1989); (b) M. Copel, M.C. Reuter, E. Kaxiras, and R.M. Tromp, Phys. Rev. Lett. 63, 632 (1989); (c) R.M. Tromp and M.C. Reuter, Phys. Rev. Lett. 68, 954 (1992); (d) N. Grandjean, J. Massies, and V.H. Etgens, Phys. Rev. Let. 69, 796 (1992); (e) H. A. van der Vegt, H.M. van Pinxteren, M. Lohmeier, E. Vlieg, and J.M.C. Thomton, Phys. Rev. Lett. 68, 3335 (1992); (f) S. Esch, M. Hohage, T. Michely, and G. Comsa, Phys. Rev. Lett. 72, 518 (1994).

[2] W.F. Egelhoff, Jr. et al, J. Appl. Phys. 80, 5183 (1996); W.F. Egelhoff, Jr., P.J. Chen, C.J. Powell, M. D. Stiles, and R.D. McMichael, J. Appl. Phys. 79, 2491 (1996).

[3] G.L. Kellogg and P.J. Feibelman, Phys. Rev. Lett. 64, 3143 (1990); C.L. Chen and T.T. Tsong, Phys. Rev. Lett. 64, 3147 (1990).

[4] D.-S. Lin, E. S. Hirschorn, T.-C. Chiang, R. Tsu, D. Lubben, and J.E. Green, Phys. Rev. B 45, 3494 (1992); J.E. Vasek, Z. Zhang, C.T. Salling, and M.G. Lagally, Phys. Rev. B 51, 17207 (1995).

[5] G.L. Kellogg, Surf. Sci. Rep. 21, 1 (1994); G. Ehrlich and F. Watanabe, Langmuir 7, 2555 (1991); T.T. Tsong, Atom-Probe Field Ion Microscopy (Cambridge University Press, Cambridge, 1990).

[6] E.W. Müller and T.T. Tsong, Field Ion Microscopy, Principles and Applications (American Elsevier, New York, 1969).

[7] G. L. Kellogg, Phys. Rev. Len. 70, 1631 (1993).

[8] G.L. Kellogg, Phys. Rev. B 55, 7206 (1997).

[9] K. Christmann, G. Ert, and T. Pignet, Surf. Sci. 54, 365 (1976); A.M. Bard, H. Ibach, and H.D. Bruchmann, Surf. Sci. 88, 384 (1979); L.H. Richter and W. Ho, Phys. Rev. B 36, 9797 (1987); J.R. Engstrom, W. Tsai, and W.H. Weinberg, J. Chem. Phys. 87. 3104 (1987).

[10] K.D. Rendulic, G. Anger, and A. Winkler, Surf. Sci. 208, 404 (1989); Y. Kim, H. C. Peebles, and J. M. White, Surf. Sci. 114, 363 (1982).

[11] G.L. Kellogg, A.F. Wright, and M.S. Daw, J. Vac. Sci. Technol. A 9, 1757 (1991).

[12] M. Scheffler, B.D. Yu, C. Ratsch, and P. Ruggerone, Abstr. Pap. Am. Chem. Soc. 313, 445 (1997). 


\title{
Hydrogen promotion of surface self-diffusion on $\mathrm{Rh}(100)$ and $\mathrm{Rh}(311)$
}

\author{
G. L. Kellogg \\ Sandia National Laboratories, Albuquerque, New Mexico 87185-1413
}

(Received 29 July 1996)

Field ion microscope observations show that the rate of surface self-diffusion for individual atoms on the (100) and (311) planes of $\mathrm{Rh}$ is significantly increased by exposure of the surface to hydrogen On $\mathrm{Rh}(100)$ admission of hydrogen at partial pressures in the $10^{-9}$-Torr range causes the onset temperature for migration of a $\mathrm{Rh}$ adatom to decrease from 290 to $240 \mathrm{~K}$. Once the adatom is mobile, its mean-square displacement is constant as a function of time. Similar exposures of hydrogen lower the onset temperanure for self-diffusion on $\mathrm{Rh}$ (311) from 180 to $120 \mathrm{~K}$. Here the mean-square displacement increases monotonically as a function of time after admission of hydrogen. The continuous increase indicates that the enhancement of the diffusion rate due to hydrogen is coverage dependent. The difference between the results for $\mathrm{Rh}(100)$ and $\mathrm{Rh}(311)$ is attributed to the higher temperatures required for self-diffusion on $\mathrm{Rh}(100)$. At the higher temperatures, a significant amount of hydrogen is thermally desorbed during the diffusion intervals and the coverage remains relatively constant as a function of time. The observed coverage dependence indicates that the mechanism of hydrogen promotion involves more than a simple lowering of the activation barrier of surface diffusion by the amachment of a hydrogen atom to a $\mathrm{Rh}$ atom. [S0163-1829(97)04212-4]

\section{INTRODUCTION}

The ability to control the evolution of surface morphology during crystal or thin-film growth by modifying elementary steps in the growth process has wide potential application in the synthesis of materials. The use of adsorbed atoms or molecules, often referred to as "surfactants," to increase the smoothness of deposited films has attracted considerable interest in recent years. ${ }^{1-9}$ Although much of the work in this area has focused on the effects of group-V and -VI surfactants such as $\mathrm{As}, \mathrm{Sb}$, and $\mathrm{Te}{ }^{3-8}$ difficulty in removing the surfactant atoms has limited the application of these materials in actual growth situations. This has stimulated efforts to use common gaseous adsorbates as surfactants, ${ }^{2.9}$ which are easily removed by thermal desorption. Hydrogen, in particular, has been shown to be a good candidate for the modification of epitaxial growth on both metal and semiconductor surfaces. ${ }^{2,10,11}$ However, our understanding of the atomicscale processes that lead to modified growth by adsorbed gases such as hydrogen is still in its infancy.

A common assumption in describing the effect of surfactants is that the chemical adsorbate in some way changes the diffusion rate of atoms involved in the growth process. For example, the formation of a smoother film may be attributed to an increase in the diffusion rate of atoms across terraces or to a reduction in the barrier for diffusion over steps. ${ }^{7,9}$ However, there are a number of steps involved in the overall process, any one of which may be subject to modification by adsorbates. To sort out the details of the different effects, it is therefore important to be able to isolate the individual steps of the growth process and determine how the adsorbate affects each one individually. In the case of atom migration across terraces, one specifically needs to know whether a given adsorbate will promote or inhibit the diffusion process.

In this study the field ion microscope (FIM) is used to investigate how hydrogen influences surface self-diffusion on $\mathrm{Rh}(100)$ and $\mathrm{Rh}(311)$. The ability of the FIM to track the motion of an individual atom as it migrates on a perfectly defined single-crystal plane ${ }^{12}$ makes it possible to separate the effect of hydrogen on the diffusion rate of a single adatom from the other steps involved in film growth. In past studies, the FIM has been successfully employed to examine the migration of individual atoms on clean surfaces and determine diffusion parameters for a variety of metal-metal combinations. ${ }^{13-15}$ Considerable care is taken in these studies to ensure that no contaminant atoms or molecules (especially hydrogen) are present on the surface during the measurements. In the experiments reported here the procedures are essentially the same, except that hydrogen is intentionally introduced to determine its effect on adatom diffusion.

There are two previous FIM studies that address the question of how hydrogen influences the mobility of metal adatoms on metal surfaces. In a study of self-diffusion on various planes of $\mathrm{Ni}$, Tung and Graham ${ }^{16}$ note that the presence of hydrogen dramatically increases the diffusion rate for $\mathrm{Ni}$ atoms on the (110), (311), and (331) planes of Ni. They find that when the Ni surfaces are prepared by thermal annealing (without hydrogen), the onset of adatom motion is observed at temperanures around $150 \mathrm{~K}$. When the surface is prepared by hydrogen-promoted field evaporation, however, adatoms are found to move freely at the base temperature of $30 \mathrm{~K}$ Even after removing the hydrogen from the system and repeated annealing and field evaporation of the substrate, residual effects of hydrogen are observed. These residual effects appear to be the most pronounced for self-diffusion on the $\mathrm{Ni}(110)$ plane.

The effect of hydrogen on. self-diffusion on W(321) is markedly different from that discussed above for diffusion on Ni surfaces. FIM studies by Casanova and Tsong ${ }^{17}$ indicate that the presence of hydrogen on the W(321) surface actually reduces the diffusion rate of a $\mathrm{W}$ adatom. In this case, the effect is relatively small: the activation energy of surface diffusion increases by only $0.05 \mathrm{eV}$ over the barrier on the clean surface. The hydrogen partial pressure used in 
these studies was $\sim 10^{-5}$ Torr and the temperature during the diffusion intervals was $\sim 300 \mathrm{~K}$. The authors indicate that under these conditions there should be a saturation coverage of hydrogen on the surface during the diffusion intervais.

Theoretical studies also indicate that the infiuence of hydrogen on metal-atom diffusion is system specific. Firstprinciples calculations by Stumpf ${ }^{18}$ show that a hydrogen atom adsorbed on top of a Be atom reduces the self-diffusion barrier on $\mathrm{Be}(0001)$ by a factor of 3 . A strong $\mathrm{H}$-Be bond weakens the bond of the adatom to its surface neighbors, i.e., a "skyhook" effect. In contrast, classical-potential totalenergy calculations by Haug et al. ${ }^{19}$ predict that a hydrogen atom acts as weak trapping center for $\mathrm{Ni}$ atoms on $\mathrm{Ni}(100)$, effectively slowing down the rate of self-diffusion. The trapping is attributed to the fact that the bydrogen atoms prefer to occupy quasisubsurface-interstitial sites. This result also appears to be at odds with the experimental result for $\mathrm{Ni}$ surfaces mentioned above, although the (100) surface was not addressed specifically in the experimental study. ${ }^{16}$

To develop a clearer picture of the role of hydrogen in promoting or inhibiting surface diffusion, it is obvious that more systems need to be examined. Self-diffusion on $\mathrm{Rh}$ was chosen for this sndy for several reasons. Past FIM studies by Ayrault and Ehrlich ${ }^{20}$ provide an extensive database relating to self-diffusion on clean $R h$ surfaces. They find that diffusion on Rh(100) takes place near room temperature, whereas diffusion on $\mathrm{Rh}(311)$ sets in at much lower temperatures $(\sim 200 \mathrm{~K})$. Hence investigations on these two surfaces permit one to examine the influence of hydrogen in different ternperanure regimes. It bas also been shown that self-diffusion on $\mathrm{Rh}(100)$ proceeds by ordinary hopping-type displacements, ${ }^{21}$ as opposed to the exchange-type displacements observed for self-diffusion on the (100) surfaces of Pt (Ref. 22) and $\mathrm{Ir}^{23}$ An additional motivation for this study was to determine whether or not the presence of hydrogen changes the diffusion mode from hopping to exchange. The ability to control the mechanism by which atoms migrate across surfaces would be very useful in efforts to modify the growth of crystals and thin films.

\section{EXPERIMENTAL DETAILS}

The experimental procedures used in FIM investigations of single-arom surface diffusion are described in detail in several recent review articles. ${ }^{13-15}$ A brief overview of these procedures is given here to aid in the discussion of the observations. In a "standard" FIM surface diffusion study a field emitter surface is cleaned by a combination of annealing, ion sputtering, and field evaporation. An image of the surface is obtained by applying a high electric field to the sample in the presence of an inert gas (e.g., He or Ne). Images are recorded with the sample at $77 \mathrm{~K}$. Individual atoms are deposited on the crystal plane of interest by heating a wire coil to a temperarure near the melting point of the metal. Atoms deposited on low index crystal planes appear as high-contrast image spots on a uniformily dark background. Motion of the deposited atom is induced by warming the surface to a preset value for a fixed interval of time in the absence of an applied electric field. The imaging gas remains in the system during the heating interval. Images are recorded immediately following each heating period. Displace- ments of the atoms are determined from the recorded images using site-visitation maps to calibrate the distance scale. The diffusion coefficient $D$ at a given temperature is obtained from the measured mean-square displacement $\left\langle r^{2}\right\rangle$ and the time interval according to $D=\left\langle r^{2}\right\rangle / 2 n \tau$, where $n$ is the dimensionality of the random walk.

In the present experiments, a single $\mathrm{Rh}$ adatom is deposited on the crystal plane of interest with pure neon at a pressure of $2 \times 10^{-4}$ Torr in the system. A sequence of diffusion intervals is carried out to establish the mean-square displacement of the adatom in the absence of hydrogen. Without removing the neon, hydrogen is leaked into the system to a preset partial pressure. The leak valve is calibrated in control experiments (without $\mathrm{Ne}$ ) to determine the partial pressure to added hydrogen. Subsequent measurements are made with the mixture of neon and hydrogen as the imaging gas. The partial pressure of hydrogen is typically in the low $10^{-9}$-Torn range. At lower partial pressures, hydrogen has a negligible effect on the diffusing adatoms. At hydrogen partial pressures above $\sim 10^{-8}$ Torr, $\mathrm{Rh}$ adatoms field desorb from the surface during field ion imaging. ${ }^{24}$ This point is discussed further below.

During the course of the experiments the surface is subjected alternatively to elevated temperanures (at zero electric field) and elevared electric fields (at $77 \mathrm{~K}$ ). The results (discussed below) indicate that some hydrogen is removed from the surface during the diffusion intervals at elevated temperatures by thermal desorption, but hydrogen is not removed by the electric field (typically $\sim 3.0 \mathrm{~V} / \AA$ ) during field ion imaging. This latter observation is consistent with previous studies of thermal field desorption of chemisorbed hydrogen from Rh surfaces. ${ }^{25}$ The primary effect of the electric field is on the adsorption of hydrogen. The electric field used for imaging can polarize hydrogen atoms in the gas phase and attract them to the surface thereby enhancing the arrival rate. $^{24}$ This effect. combined with the possibility of fieldinduced migration of hydrogen from the tip shank to the surface, makes it impossible to determine the actual hydrogen coverage in the present experimental setup. However, it is possible to investigate qualitatively the effects of increasing hydrogen coverage by examining the mobility of the adatom as a function of ime, keeping the hydrogen partial pressure constant.

A series of field ion micrographs illustrating the general procedure used in this investigation is shown in Fig. 1. Figure $\mathrm{l}$ (a) shows a single $\mathrm{Rh}$ adatom on $\mathrm{Rh}(100)$ imaged in pure $\mathrm{Ne}$ at $77 \mathrm{~K}$. Figure 1 (b) shows the same adatom after a 30 -sec heating interval at a temperature of $275 \mathrm{~K}$ in pure Ne. Measurements of the adatom's coordinates indicate that the location of the adatom is the same in Figs. 1(a) and 1(b). In ten subsequent diffusion intervals at $275 \mathrm{~K}$ with only Ne in the system, the position of the atom did not change. Figure 1(c) shows the same adatom after a hearing interval during which $2 \times 10^{-9}$ Torr of hydrogen was added to the imaging gas. Two effects of the hydrogen are observed. First, the presence of hydrogen in the imaging gas changes the characteristics of the image spot associated with the $\mathrm{Rh}$ adatom. In Fig. 1(c) the image spot appears larger and with less contrast than the spot in Figs. 1(a) and 1(b). This can be arribured to field-adsorbed hydrogen atoms as discussed below. ${ }^{24}$ More importantly, the addition of hydrogen causes the ada- 


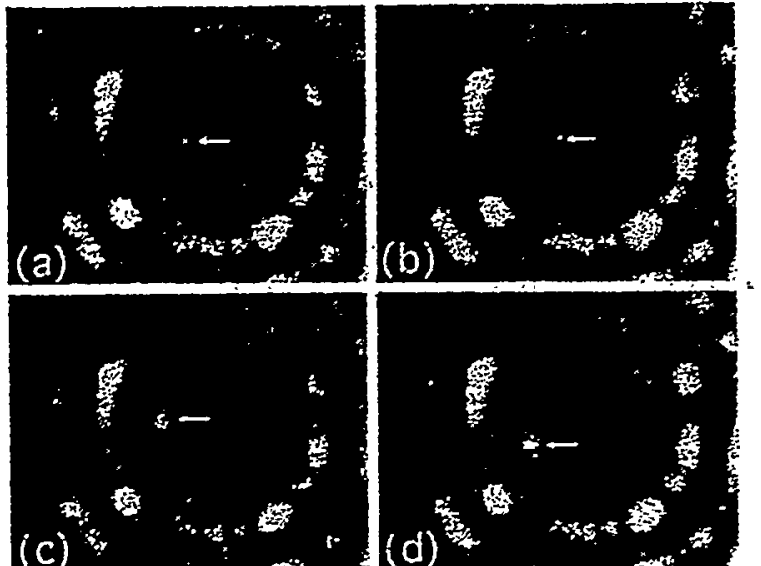

FIG. 1. Field ion microscope images showing the diffusion of an individual $\mathrm{Rh}$ adatom on $\mathrm{Rh}(100)$. Between each photograph the sample was warmed from its base temperature of $77 \mathrm{~K}$ to $275 \mathrm{~K}$. The introduction of hydrogen between (b) and (c) induces adatom mobility as discussed in the text.

tom to make a noticeable displacement. As discussed later, this is the result of chemisorbed hydrogen on the surface. Figure I(d) shows the adatom after an additional beating interval of $275 \mathrm{~K}$. Again the posicion of the adatom has changed significantly. From over 100 observations following heating intervals at $275 \mathrm{~K}$ with hydrogen present, the meansquares displacement of the atom is found to be 7.3 $\pm 1.7 \AA^{2}$. This corresponds to approximately one nearestneighbor displacement per diffusion interval. Thus the addition of hydrogen causes the initially immobile atom to migrate at an easily detectable rate.

From the orientation of the map of sites that the atom visits as it moves across the surface, it is determined that the mechanism of diffusion is ordinary bridge hopping, not the exchange mechanism found for self-diffusion on (100) surfaces of $\mathrm{Pt}$ and Ir. ${ }^{22.23}$ Since self-diffusion on $\mathrm{Rh}(100)$.in the absence of hydrogen also takes place by ordinary hopping, this result indicates that the presence of hydrogen does not change the diffusion mechanism.

\section{RESULTS}

\section{A. Self-diffusion on $\mathbf{R h}(\mathbf{1 0 0})$}

Figure 2 shows a plot of the measured mean-square displacement for a $\mathrm{Rh}$ adatom on $\mathrm{Rh}(100)$ as a function of time after admission of approximately $3 \times 10^{-9}$ Torr hydrogen to the vacuum chamber. The diffusion intervals are $30 \mathrm{sec}$ in length at a temperature of $275 \mathrm{~K}$. The bin size for each data point is 20 diffusion intervals (i.e., 20 displacements were squared and averaged for each point plotted). At a temperanure of $275 \mathrm{~K}$ and with the hydrogen present, the meansquare displacement is in the range 5-10 $\AA^{2}$, corresponding to approximately one nearest-neighbor hop per diffusion interval. From Fig. 2 it is apparent that the mean-square displacement is essentially constant as a function of time. As discussed later, this result is markedly different from that found for self-diffusion on $\mathrm{Rh}(311)$.

Figure 3 shows how the mean-square displacement for

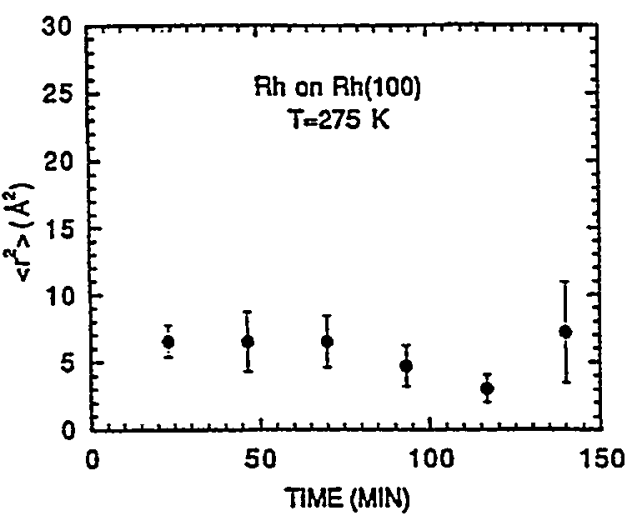

FIG. 2. Mean-square displacement $\left\langle r^{2}\right\rangle$ of a $\mathrm{Rh}$ adatom on $\mathrm{Rh}(100)$ in the presence of hydrogen remains essentially constant as a function of time.

self-diffusion on $\mathrm{Rh}(100)$ changes when the partial pressure of hydrogen is increased. The temperature during the diffusion intervals in this experiment is $280 \mathrm{~K}$. At time $t=0$ hydrogen at a partial pressure of $1 \times 10^{-9}$ Torr is admitted. Over a period of $90 \mathrm{~min}$, the mean-square displacement increases from a negligible value to $\sim 2 \AA^{2}$. When additional hydrogen is added (approximately $1 \times 10^{-9}$ Torr), the meansquare displacement increases to $\sim 4 \AA^{2}$. With the addition of even more hydrogen (approximately $2 \times 10^{-9}$ Torr) the mean-square displacement jumps to $13 \AA^{2}$. Thus an increase in hydrogen partial pressure increases the mobility of the adatom. The results shown in Fig. 4 suggest that the magnitude of each increase tends to diminish over a period of time, but the staristics are insufficient to establish the trend conclusively.

Figure 4 shows the temperature dependence of the meansquare displacement for self-diffusion on $\mathrm{Rh}(100)$ plotted in Arrhenius form for both clean and hydrogen-exposed surfaces. Qualitatively, one notes that the mobility of the adatom increases monotonically as the temperanure is increased for both cases, but the rate of increase is much less for the hydrogen-exposed surface. Figure 4 also indicates that a linear relationship is followed in the Arrhenius plots for both

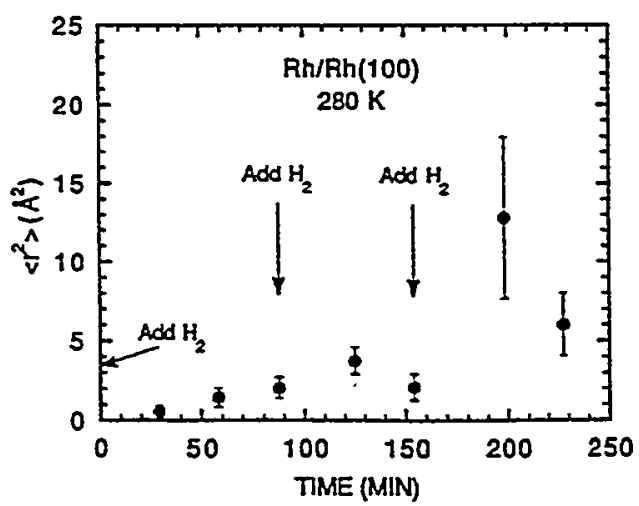

FIG. 3. Mean-square displacement $\left\langle r^{2}\right\rangle$ of a Rh adntom on $\mathrm{Rh}(100)$ increases upon addition of hydrogen to the background gas. 


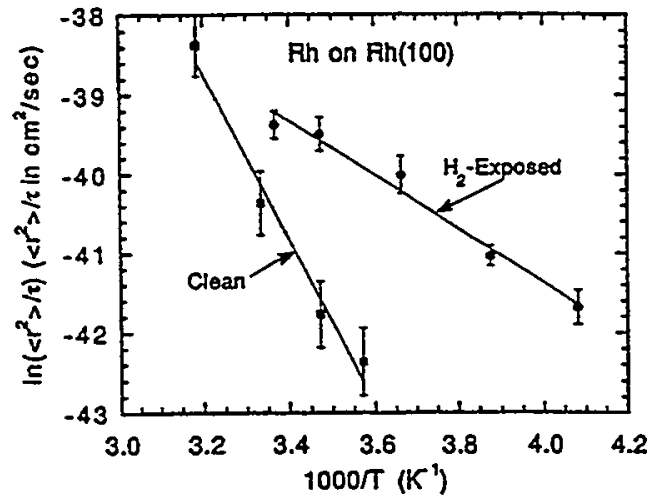

FIG. 4. Temperanure dependence of the mean-square displacement $\left\langle r^{2}\right\rangle$ for self-diffusion on $\mathrm{Rh}(100)$ plotted in Arrhenius form for clean and hydrogen-exposed surfaces. The presence of hydrogen leads to a large (apparent) reduction in the activation energy and Armenius prefactor.

situations. The activation energy and Arrhenius prefactor determined for self-diffusion on the clean surface $(0.89 \mathrm{eV}$ and $4 \times 10^{-3} \mathrm{~cm}^{2} / \mathrm{sec}$ ) agree with previous measurements by Ayrault and Ehrlich $\left(0.88 \mathrm{eV}\right.$ and $\left.1 \times 10^{-3} \mathrm{~cm}^{2} / \mathrm{sec}\right)$. For the hydrogen-exposed surface the activation energy and prefactor are both exceptionally low $(0.29 \mathrm{eV}$ and 1 $\times 10^{-12} \mathrm{~cm}^{2} / \mathrm{sec}$ ). It should be noted, however, that the results of such an analysis are misleading because, as discussed below, the coverage of hydrogen is dependent on the surface temperature during the heating interval. At higher temperarures, the coverage of hydrogen is lower. Since the mobility of the adatom is dependent on hydrogen coverage, the linear relationship observed in the Arhenius plot for the hydrogen exposed surface is most likely fortuitous and the extracted diffusion parameters cannot be interpreted in the conventional way.

\section{B. Self-diffusion on $\mathbf{R h}(311)$}

The time dependence of the mean-square displacement for self-diffusion on $\mathrm{Rh}(311)$ in the presence of hydrogen is markedly different from that for self-diffusion on $\mathrm{Rh}(100)$. Figure 5 shows the results for two separate experiments with different initial hydrogen exposures. The plots show the measured mean-square displacement during 10-sec diffusion intervals at $140 \mathrm{~K}$. Prior to admission of hydrogen, the adatom is immobile. After hydrogen is added to the background gas, the adatom remains immobile for 10-15 diffusion intervals. The mean-square displacement then increases monotonically as a function of time. The curves drawn through the data points are fits to a power-law dependence. The exponents obtained from these fits are approximately 2 , indicating a square dependence of the mean-square displacement with time (i.e., a linear dependence of the root-mean-square displacement with time).

Qualitatively, these results provide compelling evidence that hydrogen promotion of surface diffusion is indeed coverage dependent. Quantitatively, however, one cannot determine the actual coverage dependence of the mean-square displacement from the measurements. Although the thermal desorption studies mentioned below indicate that the cover-

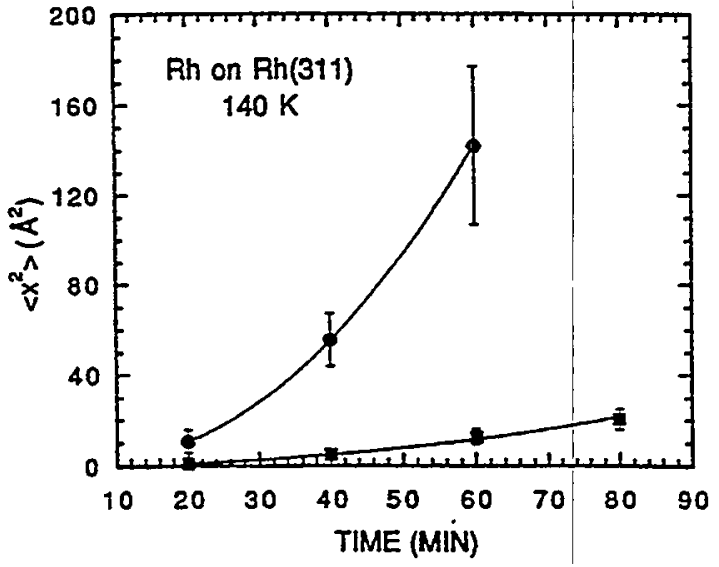

FIG. 5. Mean-square displacement $\left\langle x^{2}\right\rangle$ of a Rh adatom on $\mathrm{Rh}(311)$ in the presence of hydrogen increases rapidly as a function of time. The two curves are for two different hydrogen exposures at $140 \mathrm{~K}$. Both curves fit a power-law dependence with an exponent of -2 , indicaring a square dependence of the mean-square displacement with time.

age should increase linearly as a function of time, one must also consider the field enhancement of the supply from the gas phase during field-ion imaging as well as an additional supply due to hydrogen diffusion along the tip shank. ${ }^{24} \mathrm{Di}$ rect knowledge of the time dependence of these effects is not available, making a direct conversion of the measured time dependence into a coverage dependence beyond the scope of this study.

The temperature dependence of adatom mobility for selfdiffusion on $R h(311)$ in the presence of hydrogen was also investigated. $A s$ in the case of self-diffusion on $R h(100)$, the mean-square displacement increases with increasing temperanure. However, because the mean-square displacement also increases as a function of time as discussed above, it is not possible to determine quantitatively the change in meansquare displacement as a function of temperanure at a constant hydrogen coverage.

\section{DISCUSSION}

A general discussion of hydrogen promotion of selfdiffusion on $\mathrm{Rh}(100)$ and $\mathrm{Rh}(311)$ is facilitated by a brief review of past temperature-programmed desorption (TPD) investigations of hydrogen on these crystal surfaces. On $\mathrm{Rh}(100) \mathrm{Kim}$, Peebles, and White ${ }^{26}$ find that deuterium adsorbs dissociatively with an initial sticking coefficient of $0.53 \pm 0.05$. At low coverages corresponding to $0.05 \mathrm{~L}$ (1 $\mathrm{L}=10^{-6}$ Torr sec), the maximum of the TPD peak (taken at a heating rate of $25 \mathrm{~K} / \mathrm{sec}$ ) occurs at $361 \mathrm{~K}$ As the exposure increases. the peak maximum temperanure decreases, indicating second-order desorption kinetics. For exposures between 0.5 and $1 \mathrm{~L}$, a shoulder appears at about $260 \mathrm{~K}$. At exposures of $0.8-1.0 \mathrm{~L}$, a low-temperanure desorption peak also appears and the high-temperature peak broadens on its low-temperature side. Measurements of the areas under the TPD curves indicate that the coverage of denterium versus exposure is linear up to about $0.5 \mathrm{~L}$ of deuterium. The thermal desorption kinetics for hydrogen on $\mathrm{Rh}(100)$ are pre- 
sumed to be identical to those for deuterium

On Rh(311) TPD measurements by Nichtl-Pecher et al. ${ }^{27}$ indicate that five distinct binding states are populated upon hydrogen exposures at $90 \mathrm{~K}$. These states originate from dissociatively adsorbed hydrogen as proven by isotope exchange experiments. The value of the initial sticking coefficient is $\sim 0.25$. The highest-temperature (lowest-coverage) peak is at about $350 \mathrm{~K}$. A large low-temperanure peak ( - 175 $\mathrm{K})$ begins to grow in at exposures above $0.6 \mathrm{~L}$. Two additional peaks appear at intermediate exposures. Above exposures of $1 \mathrm{~L}$, an even lower-temperature $(\sim 125 \mathrm{~K})$ peak appears.

These TPD studies make it clear that hydrogen adsorbs dissociatvely on both the (100) and (311) surfaces of $\mathrm{Rh}$ with a relatively high sticking coefficient. It is therefore reasonable to assume that the observed promotion of self-diffusion on both surfaces is due to adsorbed atomic hydrogen. This being the case, it is somewhat surprising to find that the time dependence of the measured mean-square displacement is qualitatively different for the two different substrates. On $\mathrm{Rh}(100)$ the mean-square displacement of a $\mathrm{Rh}$ adatom increases upon exposure of the surface to hydrogen, but then remains constant as a function of time after exposure (Fig. 2). At first glance, this suggests that the hydrogen promotion effect is independent of coverage because the coverage is expected to increase as a function of time with a constant pressure in the background. On $\mathrm{Rh}(311)$, however, the meansquare displacement increases dramatically as a function of time (Fig. 5). This indicates a strong dependence of the diffusion rate on hydrogen coverage.

The difference in the time dependence for the two crystal planes can be explained by the difference in the temperanure required to initiate self-diffusion on the two surfaces. On the clean $R h(100)$ surface the onset of self-diffusion occurs at temperatures near $300 \mathrm{~K}$. Exposure of the surface to hydrogen lowers the onset temperature to $\sim 260 \mathrm{~K}$. In this temperature range, the thermal desorption studies mentioned above indicate that a substantial fraction of adsorbed hydrogen will thermally desorb from the surface during the heating intervals. Hydrogen is readsorbed during the time between the hearing intervals (i.e., during field ion imaging). A steady-state hydrogen coverage is established and the diffusion rate remains constant as a function of time.

This argument explains why the self-diffusion rate on $\mathrm{Rh}(100)$ increases when additional hydrogen is added to the system (as shown in Fig. 3). The higher partial pressure causes an increase in hydrogen coverage. The subsequent decreases suggested by the data shown in Fig. 3 can be explained by thermal desorption of hydrogen over the successive heating intervals, i.e., it takes a number of desorption cycles before a steady state is reestablished. As indicated earlier, this explanation is also consistent with the observed temperature dependence of the mean-square displacement on $\mathrm{Rh}(100)$ and the exceptionally low (apparent) diffusion parameters (Fig. 4). Thermal desorption of hydrogen during the heating intervals causes the steady-state coverage of hydrogen to be higher at successively lower temperatures. As the temperature is lowered in the experiments, the rate of diffusion is enhanced due to the higher hydrogen coverage. In an Arrhenius plot, this has the effect of reducing both the slope and the prefactor leading to unrealistic values of the diffusion parameters.

On $\mathrm{Rh}(311)$ the situation is quite different. Here the temperature range of the experiments is $120-160 \mathrm{~K}$. According to the thermal desorption studies, ${ }^{27}$ little hydrogen will be thermally desorbed at these temperatures during the diffusion intervals. This allows the hydrogen coverage to build up over a period of time and, as the coverage builds up, the diffusion rate increases. One therefore observes a marked increase in the measured mean-square displacement as a function of time.

It is interesting that the mobility of an adatom exposed to hydrogen in the low $10^{-9}$-Torr range increases as a function of time for over $1 \mathrm{~h}$, but the adatom does not field desorb. Yet, if the partial pressure of hydrogen is raised to $\sim 10^{-8}$ Torr the adatom immediately desorbs. Apparently, the desorption of the adatom is promoted by field-adsorbed hydrogen (weakly bound hydrogen held on the surface by polarization forces due to the applied electric feld ${ }^{24}$ ) from the gas phase, whereas the mobility of the adatom is promoted by chemisorbed hydrogen on the surface. When the surface is heated, field-adsorbed hydrogen is removed, but chemisorbed hydrogen remains. This field adsorbed hydrogen also explains the change in image spor size as mentioned above.

It is imporant to emphasize that the diffusion rate is coverage dependent for self-diffusion on both $R h(100)$ and $\mathrm{Rh}(311)$, but the coverage dependence manifests itself as a time dependence in the measured mean-square displacement only in the case of $\mathrm{Rh}(311)$. On $\mathrm{Rh}(100)$ the coverage dependence is inferred from the increase in the diffusion rate upon the addition hydrogen to the background and on the temperature dependence of the mean-square displacement as discussed above. From a mechanistic standpoint, the observation of a coverage-dependent effect in the promotion of diffusion by hydrogen rules out a simple skybook effect ${ }^{13}$ (i.e., a weakening of the metal-metal bonds by a strong $\mathrm{H}$-metal bond). In this case, one would expect a single-step increase in the diffusion rate corresponding to when a hydrogen atom anaches itself to the $\mathrm{Rh}$ adatom. The monotonic increase in the diffusion rate with hydrogen coverage indicates that if a skyhook effect exists at all, it is a transient effect, i.e., once a displacement occurs, the hydrogen is no longer bound to the diffusing atom.

The results of this study are also inconsistent with the conclusions of theoretical studies for the effect of hydrogen on the diffusion rate of $\mathrm{Ni}$ atoms on $\mathrm{Ni}(100) .{ }^{19}$ The calculations predict that the presence of hydrogen should decrease the rate of self-diffusion on $\mathrm{Ni}(100)$, whereas the result reported here for $\mathrm{Rh}(100)$ indicates that the presence of hydrogen increases the rate. This suggests either that the effect of hydrogen is totally different for self-diffusion on $\mathrm{Rh}(100)$ and $\mathrm{Ni}(100)$ or, perhaps, the calculations, which involve only one hydrogen atom per metal adatom, are not an adequate representation of the experimental simation.

Having ruled out a simple skyinook effect as the mechanism of promotion, it is natural to speculate on alternative possibilities. It could be that the diffusion barrier is lowered by an interaction similar to a skyhook effect, but in the process of making a displacement, the hydrogen atom detaches itself from the adatom. Another possibility is that the promotion effect is not due to a direct interaction of the hydrogen 


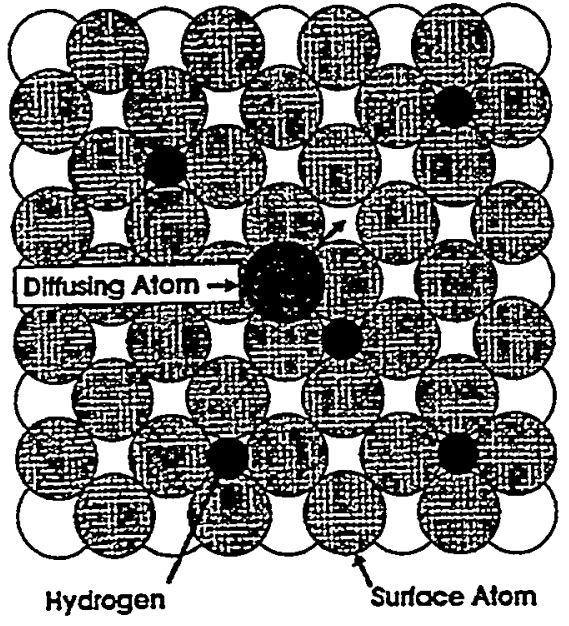

FIG. 6. Schematic drawing of a $\mathrm{Rh}$ atom on $\mathrm{Rh}(100)$, indicating a possible mechanism for the promotion of diffusion by the presence of hydrogen.

with the diffusing atom, but due to the interaction of the hydrogen with the surface atoms in the vicinity of the adatom. A plausible scenario for the case of self-diffusion on fcc(100) surfaces is shown in Fig. 6. When a hydrogen atom occupies a fourfold hollow site next to the adatom, the interaction of the hydrogen with the substrate atoms may reduce the diffusion barrier for the adatom by either weakening its binding to the substrate at the equilibrium position or lowering the energy of the saddle point at one of the bridge sites. Previous studies of hydrogen diffusion on other metal surfaces indicate that at temperatures high enough to induce motion of a $\mathrm{Rh}$ adatom, hydrogen atoms will be highly mobile. ${ }^{28}$ Once the hydrogen atom promotes a displacement, it moves quickly away from the vicinity of the adatom. In order for the adatom to displace again, another hydrogen atom must come along. As the coverage increases, the probability of a hydrogen atom finding itself next to the adatom becomes higher, leading to a coverage-dependent promotion effect. The coverage dependence would be enhanced if hydrogen atoms were required at two of the neighboring sites instead of just one.

Obviously, the above mechanisms cannot be verified by the present experiments. To gain further insight into the pro- motion mechanism, a method is needed to determine the actual coverage of hydrogen during the experiment. This may be possible by the measurement of field emission workfunction changes carried out during the course of the field ion microscope studies. For a given dipole moment and a given tip radius, the change in work function due to the adsorption is directly related to the coverage of the adsorbed species. ${ }^{29}$ Unfortunately, such measurements are not feasible in our current experimental setup. Further insight into the promotion mechanism could also be obtained from detailed calculations of the energetics associated with a hydrogen atom in the vicinity of a metal adatom.

\section{CONCLUSION}

The results of this investigation lead to three important conclusions: (i) the adsorption of hydrogen on both the (100) and (311) planes of $\mathrm{Rh}$ significantly enhances the rate of surface self diffusion, (ii) the promotion effect is dependent on the coverage of hydrogen, and (iii) the presence of hydrogen does not change the diffusion mechanism from hopping to exchange. With regard to the use of hydrogen as a surfactant as discussed in the Introduction, the second conclusion is significant in that it suggests the possibility of a controllable adjustment in the diffusion rate of atoms across terraces, of one of the elementary steps in the growth process. Although the inability to characterize the actual coverage of hydrogen in the experiments makes it difficult to interpret the results in a quantitative fashion, the observed time and temperature dependences allow us to eliminate a simple skyhook effect as a mechanism of promotion and make some speculations as to what the mechanism might be. In experiments planned for the near future, the effect of hydrogen on diffusion for a system that the exchange mechanism is energetically favorable [e.g., self-diffusion on $\mathrm{Pt}(100)]$, will be examined to determine if hydrogen changes the preferred mechanism in the reverse direction, i.e., from exchange to hopping.

\section{ACKNOWLEDGMIENTS}

It is a pleasure to acknowledge many fruitful discussions with Brian Swartzentruber throughout the course of this investigation. This work is supported by the U.S. Deparment of Energy under Contract No. DE-AC04-94AL85000.
${ }^{1}$ D. A. Steigerwald, I. Jacob, and W. F. Egelhoff, Ir., Surf. Sci. 202, 472 (1988).

${ }^{2}$ W. F. Egelhoff. Jr. and D. A. Steigerwald, J. Vac. Sci. Technol. A 7, 2167 (1989).

${ }^{3}$ M. Copel, M. C. Reuter, E. Kaxiras, and R. M. Tromp, Phys. Rev. Let. 63, 632 (1989).

${ }^{4}$ M. Horn-von Hoegen. F. K. LeGoues, M. Copel, M. C. Reuter, and R. M. Tromp. Phys. Rev. Lett. 67, 1130 (1991).

${ }^{5}$ R. M. Tromp and M. C. Reuter, Phys. Rev. Lert 68, 954 (1992).

${ }^{6} \mathrm{~N}$. Grandjean. J. Massies, and V. H. Etgens, Phys. Rev. Lett. 69. 796 (1992).

${ }^{7}$ H. A. van der Vegt, H. M. van Pinxteren. M. Lohmeier, E. Vlieg. and J. M. C. Thomton. Phys. Rev. Lett. 68. 3335 (1992).

${ }^{8}$ G. Rosenfeld. R. Servaty. C. Teichert. B. Poelsema, and G. Comsa. Phys. Rev. Lett. 71.895 (1993).

${ }^{9}$ S. Esch. M. Hohnge. T. Michely, and G. Comsa. Phys. Rev. Lett. 72, 518 (1994).

${ }^{10}$ D.-S. Lin. E. S. Hirschom, T.-C. Chiang, R. Tsu. D. Lubben. and J. E. Green, Phys. Rev. B 45, 3494 (1992).

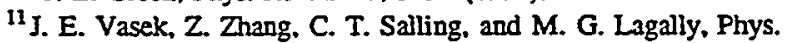
Rev. B 51, 17207 (1995).

${ }^{12}$ G. Ehrlich and F. G. Hudda, J. Chem. Phys. 44, 1039 (1966).

${ }^{13} \mathrm{G}$. Enrlich and F. Watanabe, Langmuir 7, 2555 (1991).

${ }^{14}$ T. T. Tsong. Atom-Probe Field Ion Microscopy (Cambridge Uni- 
versity Ptess, Cambridge, England, 1990), pp. 202-265.

${ }^{15}$ G. L. Kellogg, Surf. Sci. Rep. 21, 1 (1994).

${ }^{16}$ R. T. Tung and W. R. Graham, Surf. Sci. 97.73 (1980).

${ }^{17}$ R. Casanova and T. T. Tsong, Surf. Sci. 94, L179 (1980).

${ }^{18}$ R. Stumpf, Phys. Rev. B 53, R4253 (1996).

${ }^{19} \mathrm{~K}$. Haug, Z. Zhang, C. F. Walkers, D. M. Zehner, and W. E. Plummer (unpublished).

${ }^{20} \mathrm{G}$. Ayrault and G. Ehrlich, J. Chem. Phys. 60, 281 (1974).

${ }^{21}$ G. L. Kellogg, Phys. Rev. Letr. 73, 1833 (1994).

${ }^{22}$ G. L. Kellogg and P. J. Feibelman, Phys. Rev. Lett. 64, 3143 (1990).

${ }^{23}$ C. L. Chen and T. T. Tsong, Phys. Rev. Lett. 64, 3147 (1990).
${ }^{24}$ E. W. Müller and T. T. Tsong, Field Ion Microscopy, Principles and Applications (American Elsevier, New York. 1969).

${ }^{25}$ N. Ernst. J. H. Block, H. I. Kreuzer, and X. Ye, Phys. Rev. Lett. 71. 891 (1993).

${ }^{26}$ Y. Kim, H. C. Peebles, and J. M. White, Surf. Sci. 114, 363 (1982).

${ }^{27}$ W. Nichtl-Pecker, W. Stammlet, K. Heinz, and K. Müller, Phys. Rev. B 43, 6946 (1991).

${ }^{28}$ R. Gomer, Rep. Prog. Phys. 53, 917 (1990).

${ }^{29} \mathrm{R}$. Gomer, Field Emission and Field Ionization (Harvard University Press, Cambridge, MA, 1961). 
Distribution:

1 MS-0188

4 MS-1413

1 MS-1413

1 MS-1415

1 MS-1421

1 MS-1421

1 MS-1421

1 MS- 1427

1 MS-9161

1 MS-9161

1 MS-9161

1 MS-9018

2 MS-0899

1 MS-0612

LDRD Office

P. J. Feibelman, 1114

T. A. Michalske, 1115

J. A. Floro, 1112

N. D. Shinn, 1114

G. L. Kellogg, 1114

B. S. Swartzentruber, 1114

S. T. Picraux, 1100

R. Q. Hwang, 8721

N. C. Bartelt, 8721

J. C. Hamilton, 8721

Central Technical Files, 8940-2

Technical Library, 4916

Review \& Approval Desk, 4912 for DOE/OSTI 